\title{
Stereocontrolled Synthesis of Dafachronic Acid A, the Ligand for the DAF-12 Nuclear Receptor of Caenorhabditis elegans
}

\author{
Simon Giroux and E. J. Corey* \\ Department of Chemistry and Chemical Biology, Harvard University, 12 Oxford Street \\ Cambridge, Massachusetts 02138

\section{Supporting Information}

1. Materials and Methods. Unless stated otherwise, reactions were performed in flame-dried glassware under a positive pressure of nitrogen using freshly distilled dry solvents. Commercial grade reagents and solvents were used without further purification except as indicated below. $\mathrm{MeOH}$ was distilled over $\mathrm{CaSO}_{4}$. Dichloromethane was distilled from calcium hydride. Toluene, $\mathrm{Et}_{2} \mathrm{O}$ and $\mathrm{THF}$ were purified by Seco Solvent Systems. Thin-layer chromatography (TLC) was performed using E. Merck silica gel $60 \mathrm{~F}_{254}$ precoated plates $(0.25 \mathrm{~mm})$. Flash chromatography was performed using Baker silica gel (40 $\mu \mathrm{m}$ particle size). ${ }^{1} \mathrm{H}$ NMR spectra were recorded on Varian Mercury 400 (400 MHz) or Unity/INOVA $500(500 \mathrm{MHz})$ spectrometers and chemical shifts are reported in ppm from tetramethylsilane with the solvent resonance as internal standard $\left(\delta 7.26 \mathrm{ppm}\right.$ for $\left.\mathrm{CDCl}_{3}\right) \cdot{ }^{13} \mathrm{C}$ NMR spectra were recorded on Varian Mercury 400 (100 MHz) or Unity/INOVA 500 (125 $\mathrm{MHz}$ ) spectrometers with proton decoupling. Chemical shifts are reported in ppm from tetramethylsilane with the solvent as internal $\left(\delta 77.16 \mathrm{ppm}\right.$ for $\mathrm{CDCl}_{3}$ ). IR spectra were recorded on Avatar 360 FT-IR spectrometer. Low-resolution and high-resolution mass spectral analyses were performed at the Harvard University Mass Spectrometry Center. Optical rotations were measured with a Perkin-Elmer 241 polarimeter at the indicated temperature with a sodium lamp (D line, $589 \mathrm{~nm}$ ). Melting points (m.p.) are uncorrected and were recorded on a Thomas-Hoover Unimelt capillary melting point apparatus. 


\section{Experimental procedures.}

\section{$E, E$-dienoate (4)}

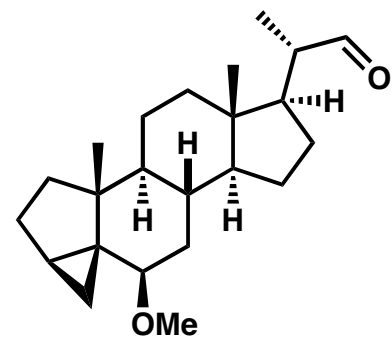

2

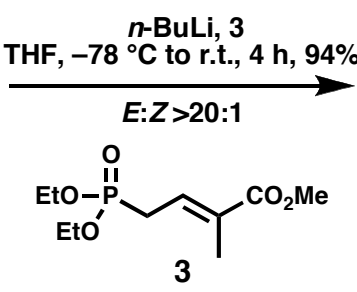

3

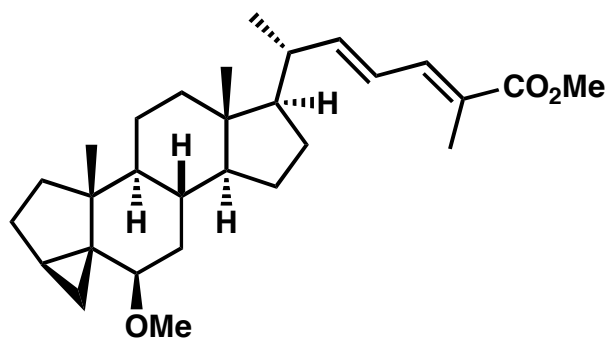

4

To a stirred solution of phosphonate $\mathbf{3}^{1}(3.11 \mathrm{~g}, 12.5 \mathrm{mmol})$ in THF (40 mL) was added $n$-BuLi (4.64 mL, $2.5 \mathrm{M}$ in hexanes, $11.6 \mathrm{mmol}$ ) at $-78^{\circ} \mathrm{C}$. The resulting bright yellow solution was stirred for $30 \mathrm{~min}$ at $-78^{\circ} \mathrm{C}$ and the aldehyde $2^{2}(2.86 \mathrm{~g}, 8.3 \mathrm{mmol})$ was slowly added via canula (20 mL, THF and $2 \times 3 \mathrm{~mL}$ rinse). The pale yellow solution was stirred at $-78^{\circ} \mathrm{C}$ for $1 \mathrm{~h}$ and warmed gradually to r.t. over $3 \mathrm{~h}$ and quenched with a saturated solution of aq. $\mathrm{NH}_{4} \mathrm{Cl}(50 \mathrm{~mL})$. The aqueous phase was extracted with $\mathrm{Et}_{2} \mathrm{O}(3 \times 50 \mathrm{~mL})$ and the organic phase was dried over $\mathrm{Na}_{2} \mathrm{SO}_{4}$. After evaporation, the residue was purified by flash chromatography $(95: 5 \rightarrow 9: 1$; hexanes/EtOAc) to give the dienoate $4(3.43 \mathrm{~g}, 94 \%, E / Z>$ 20:1) as a white solid. $[\alpha]_{\mathrm{D}}+42.7\left(c\right.$ 0.11, $\left.\mathrm{CHCl}_{3}\right)$; m.p. 96-98 ${ }^{\circ} \mathrm{C} ;{ }^{1} \mathrm{H}$ NMR $(500 \mathrm{MHz}$, $\left.\mathrm{CDCl}_{3}\right) \delta 7.13(\mathrm{~d}, 1 \mathrm{H}, J=11.2 \mathrm{~Hz}), 6.24(\mathrm{dd}, 1 \mathrm{H}, J=11.2$ and $15.1 \mathrm{~Hz}), 5.92(\mathrm{dd}, 1 \mathrm{H}, J=8.8$ and $15.1 \mathrm{~Hz}), 3.73(\mathrm{~s}, 3 \mathrm{H}), 3.31(\mathrm{~s}, 3 \mathrm{H}), 2.76(\mathrm{~s}, 1 \mathrm{H}), 2.24(\mathrm{~m}, 1 \mathrm{H}), 1.96(\mathrm{dt}, 1 \mathrm{H}, J=3.4$ and $12.6 \mathrm{~Hz}), 1.91(\mathrm{~s}, 3 \mathrm{H}), 1.86(\mathrm{dt}, 1 \mathrm{H}, J=2.9$ and $13.6 \mathrm{~Hz}), 1.74(\mathrm{~m}, 2 \mathrm{H}), 1.71-1.44(\mathrm{~m}, 5 \mathrm{H})$, $1.41(\mathrm{~m}, 2 \mathrm{H}), 1.21(\mathrm{~m}, 3 \mathrm{H}), 1.07(\mathrm{~d}, 3 \mathrm{H}, J=6.8 \mathrm{~Hz}+1 \mathrm{H}), 1.02(\mathrm{~s}, 3 \mathrm{H}+1 \mathrm{H}), 0.85(\mathrm{~m}, 3 \mathrm{H})$, $0.74(\mathrm{~s}, 3 \mathrm{H}), 0.64(\mathrm{t}, 1 \mathrm{H}, J=4.4 \mathrm{~Hz}), 0.42(\mathrm{dd}, 1 \mathrm{H}, J=5.4$ and $8.3 \mathrm{~Hz}) ;{ }^{13} \mathrm{C} \mathrm{NMR}(125 \mathrm{MHz}$, $\left.\mathrm{CDCl}_{3}\right) \delta 169.3,149.7,139.5,124.8,123.6,82.5,56.8,56.6,55.8,51.9,48.2,43.6,43.2,40.9$, 40.3, 35.4, 35.2, 33.5, 30.7, 28.6, 25.1, 24.4, 22.9, 21.6, 20.1, 19.5, 13.3, 12.8, 12.7; IR (film, $\left.\mathrm{cm}^{-1}\right)$ 2930, 1706, 1635, 1434, 1244, 1096; MS (ES +) m/z (\%): 441.7 (100). 
$\alpha, \beta$-unsaturated acid (5)
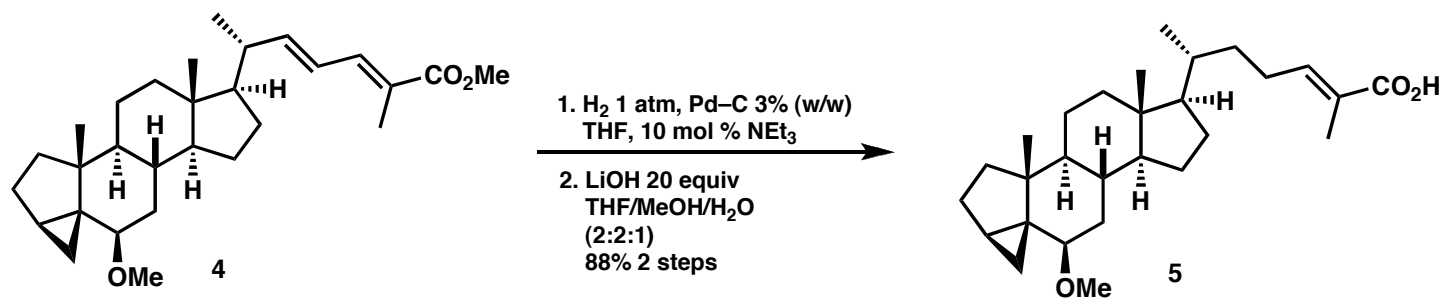

To a stirred solution of dienoate $4(1.85 \mathrm{~g}, 4.19 \mathrm{mmol})$ in THF $(40 \mathrm{~mL})$ and $\mathrm{NEt}_{3}(0.058 \mathrm{~mL}$, $0.419 \mathrm{mmol})$ was added $\mathrm{Pd} / \mathrm{C}(55 \mathrm{mg}, 3 \% \mathrm{w} / \mathrm{w})$. A hydrogen-filled balloon (1 atm) was placed over the solution and the mixture was stirred for $20 \mathrm{~min}$, by which time TLC analysis indicated that the reaction was completed. The mixture was filtered through a pad of Celite ${ }^{\circledR}$ and washed with EtOAc $(25 \mathrm{~mL})$. Evaporation gave the methyl ester of 5 that was directly submitted to hydrolysis without any further purification.

To a stirred solution of the methyl ester of 5 in $\mathrm{THF} / \mathrm{MeOH} / \mathrm{H}_{2} \mathrm{O}(2: 2: 1)(85 \mathrm{~mL})$ was added $\mathrm{LiOH}(3.51 \mathrm{~g}, 83.8 \mathrm{mmol})$ at room temperature. The resulting mixture was stirred at r.t. for $15 \mathrm{~h}$. The reaction mixture was acidified to ca. $\mathrm{pH} 2$ by addition of $1 N \mathrm{HCl}(20 \mathrm{~mL})$ and the aqueous phase was extracted with $\mathrm{CH}_{2} \mathrm{Cl}_{2}(3 \times 50 \mathrm{~mL})$. The combined organic phase were dried over $\mathrm{Na}_{2} \mathrm{SO}_{4}$ and concentrated to dryness. The residue was purified by flash chromatography (8:2 $\rightarrow 7: 3$; hexanes/EtOAc) to give the title acid $\mathbf{5}(1.59 \mathrm{~g}, 88 \% 2$ steps $)$ as a white solid. $[\alpha]_{\mathrm{D}}+41.5\left(c 0.06, \mathrm{CHCl}_{3}\right)$; m.p. $87-88{ }^{\circ} \mathrm{C} ;{ }^{1} \mathrm{H} \mathrm{NMR}\left(400 \mathrm{MHz}, \mathrm{CDCl}_{3}\right) \delta 6.89$ (t, 1H, J = 7.3 Hz), $3.32(\mathrm{~s}, 3 \mathrm{H}), 2.77(\mathrm{~s}, 1 \mathrm{H}), 2.22(\mathrm{~m}, 1 \mathrm{H}), 2.12(\mathrm{~m}, 1 \mathrm{H}), 1.97(\mathrm{~m}, 1 \mathrm{H}), 1.87$ $(\mathrm{m}, 1 \mathrm{H}), 1.83(\mathrm{~s}, 3 \mathrm{H}), 1.74(\mathrm{~m}, 3 \mathrm{H}), 1.60(\mathrm{~m}, 1 \mathrm{H}), 1.51(\mathrm{~m}, 3 \mathrm{H}), 1.41(\mathrm{~m}, 3 \mathrm{H}), 1.25(\mathrm{~m}, 2 \mathrm{H})$, 1-15-1.03 (m, 5H), $1.01(\mathrm{~s}, 3 \mathrm{H}), 0.95(\mathrm{~d}, 3 \mathrm{H}, J=6.2 \mathrm{~Hz}), 0.89-0.83(\mathrm{~m}, 4 \mathrm{H}), 0.71(\mathrm{~s}, 3 \mathrm{H})$, $0.64(\mathrm{t}, 1 \mathrm{H}, J=4.4 \mathrm{~Hz}), 0.43(\mathrm{dd}, 1 \mathrm{H}, J=5.1$ and $8.1 \mathrm{~Hz}) ;{ }^{13} \mathrm{C} \mathrm{NMR}\left(125 \mathrm{MHz}, \mathrm{CDCl}_{3}\right) \delta$ $173.5,146.0,126.8,82.6,56.8,56.7,56.3,48.2,43.6,43.0,40.4,35.9,35.4,35.2,34.7,33.5$, 30.6, 285, 25.9, 25.1, 24.3, 22.9, 21.7, 19.5, 187, 13.3, 12.4, 12.1; IR (film, $\mathrm{cm}^{-1}$ ) 2931, 1684, 1558, 1456, 1096; MS (ES +) m/z (\%): 429.5 (100). 

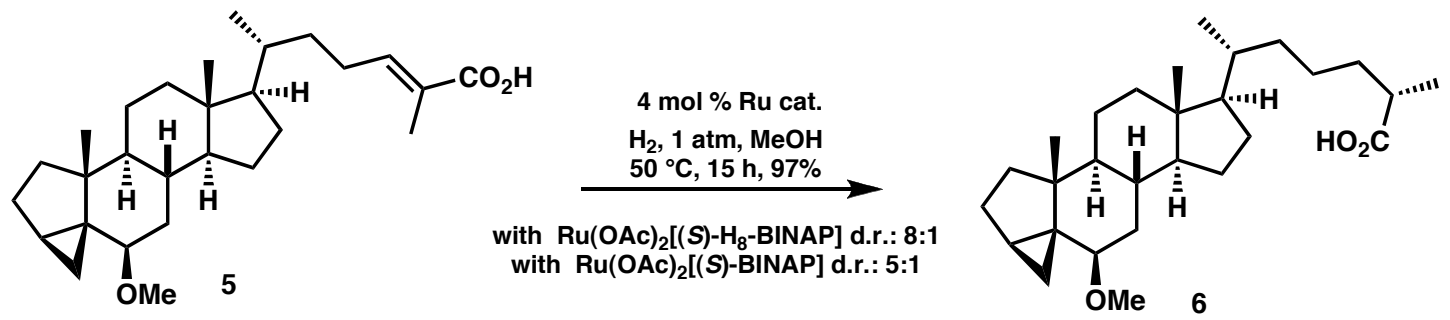

The acid 5 (833 mg, $1.94 \mathrm{mmol})$ is dissolved in $\mathrm{MeOH}$ (18 $\mathrm{mL}$, degassed with 3 freeze-pump-thaw cycle). To this solution was added a solution of $\operatorname{Ru}(\mathrm{OAc})_{2}[(S)$-BINAP] (62 $\mathrm{mg}, 0.04 \mathrm{mmol})$ in $\mathrm{MeOH}(1 \mathrm{~mL})$. The resulting yellow solution was stirred at $50{ }^{\circ} \mathrm{C}$ under $\mathrm{H}_{2}$ for $15 \mathrm{~h}$. The mixture was evaporated and the residue was purified by flash chromatography (dichloromethane/MeOH; 95:5) to give the acid 4 (814 mg, 97\%; dr.: 5:1) as a beige solid. Recrystallization with $(i \mathrm{Pr})_{2} \mathrm{O}$ affords colorless needles with d.r. $>10: 1$. The same reaction using $\mathrm{Ru}(\mathrm{OAc})_{2}\left[(S)-\mathrm{H}_{8}\right.$ - BINAP $]$ as catalyst affords the desired acid with a d.r. of $8: 1$ in $97 \%$ yield. $[\alpha]_{\mathrm{D}}+58.0\left(c 0.06, \mathrm{CHCl}_{3}\right)$; m.p. $161-162{ }^{\circ} \mathrm{C} ;{ }^{1} \mathrm{H}$ NMR $(400 \mathrm{MHz}$, $\left.\mathrm{CDCl}_{3}\right) \delta 3.31(\mathrm{~s}, 3 \mathrm{H}), 2.77(\mathrm{~s}, 1 \mathrm{H}), 2.44(\mathrm{qt}, 1 \mathrm{H}, J=6.9 \mathrm{~Hz}), 1.96(\mathrm{dt}, 1 \mathrm{H}, J=3.3$ and 12.4 $\mathrm{Hz}), 1.88(\mathrm{dt}, 1 \mathrm{H}, J=2.9$ and $13.5 \mathrm{~Hz}), 1.85-1.56(\mathrm{~m}, 5 \mathrm{H}), 1.50(\mathrm{~m}, 2 \mathrm{H}), 1.38(\mathrm{~m}, 6 \mathrm{H}), 1.24$ $(\mathrm{m}, 2 \mathrm{H}), 1.17(\mathrm{~d}, 3 \mathrm{H}, J=6.9 \mathrm{~Hz}), 1.16-0.98(\mathrm{~m}, 9 \mathrm{H}), 0.89(\mathrm{~d}, 3 \mathrm{H}, J=6.6 \mathrm{~Hz}), 0.87-0.73(\mathrm{~m}$, 4H), $0.70(\mathrm{~s}, 3 \mathrm{H}), 0.63(\mathrm{t}, 1 \mathrm{H}, J=4.4 \mathrm{~Hz}), 0.42(\mathrm{dd}, 1 \mathrm{H}, J=5.1$ and $7.7 \mathrm{~Hz}) ;{ }^{13} \mathrm{C}$ NMR $(125$ $\left.\mathrm{MHz}, \mathrm{CDCl}_{3}\right) \delta 183.1,82.7,56.7,56.4,48.2,43.6,43.0,40.5,39.6,39.5(\mathrm{~min}), 35.9,35.8$, $35.4,35.2,34.2,34.1(\mathrm{~min}), 33.5,30.6,28.5,25.1,24.3,23.9,23.8(\mathrm{~min}), 22.9,21.7,19.5$, 18.8, 17.2, 16.9(min), 13.3, 12.4.; IR (film, $\left.\mathrm{cm}^{-1}\right)$ 2933, 2866, 1733, 1700, 1558, 1456, 1096; HRMS calcd for $\mathrm{C}_{28} \mathrm{H}_{48} \mathrm{O}_{4}\left(\mathrm{M}+\mathrm{H}_{2} \mathrm{O}\right) 448.3791$ found: 448. 3770 . 

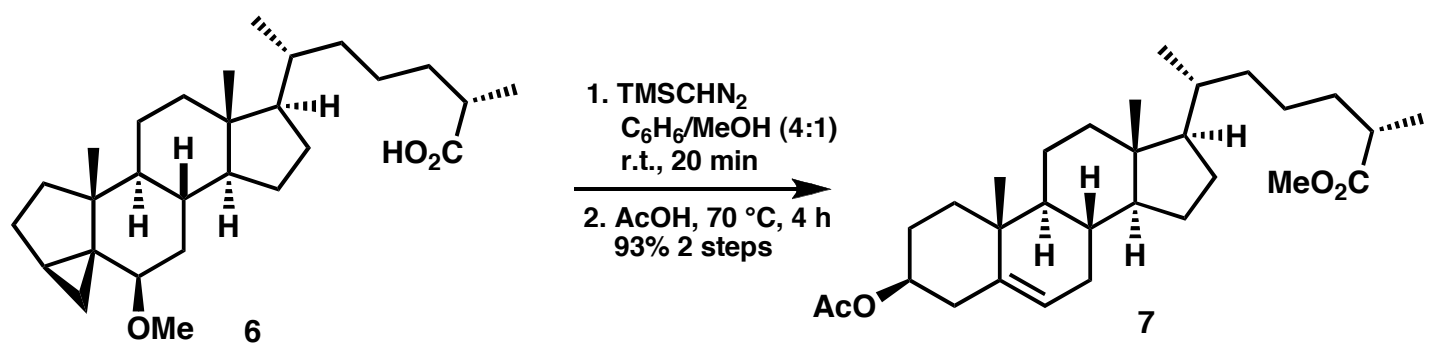

To a solution of the acid $6(1.46 \mathrm{~g}, 3.39 \mathrm{mmol})$ in $\mathrm{C}_{6} \mathrm{H}_{6} / \mathrm{MeOH}(4: 1)(34 \mathrm{~mL})$ was added $\mathrm{TMSCHN}_{2}\left(2 \mathrm{M}\right.$ in $\left.\mathrm{Et}_{2} \mathrm{O}, 2.03 \mathrm{~mL}, 4.07 \mathrm{mmol}\right)$. The resulting solution was stirred for $20 \mathrm{~min}$ at r.t. and evaporated to dryness. The residue was purified by flash chromatography (hexanes/EtOAc; 9:1) to give the ester $(1.49 \mathrm{mg}, 99 \%)$ which was submitted to the next reaction.

A solution of the above ester $(1.50 \mathrm{~g}, 3.37 \mathrm{mmol})$ in glacial AcOH $(33 \mathrm{~mL})$ was heated for $4 \mathrm{~h}$ at $90{ }^{\circ} \mathrm{C}$. The solution was cooled to r.t., diluted with $\mathrm{H}_{2} \mathrm{O}(20 \mathrm{~mL})$, and slowly neutralized with saturated aq. $\mathrm{NaHCO}_{3}$. The aqueous phase was extracted with EtOAc and the organic phase was dried with $\mathrm{Na}_{2} \mathrm{SO}_{4}$ and concentrated to dryness. The residue was purified by flash chromatography (hexanes/EtOAc; $9: 1)$ to give the $7(1.5 \mathrm{~g}, 94 \%)$ as a white solid. $[\alpha]_{\mathrm{D}}-30.0$ (c $0.06, \mathrm{CDCl}_{3}$ ); m.p. $110{ }^{\circ} \mathrm{C} ;{ }^{1} \mathrm{H}$ NMR $\left(500 \mathrm{MHz}, \mathrm{CDCl}_{3}\right) \delta 5.33(\mathrm{~s}, 1 \mathrm{H}), 4.57(\mathrm{~m}, 1 \mathrm{H}), 3.63$ (s, 3H), $2.40(\mathrm{~m}, 1 \mathrm{H}), 2.27(\mathrm{~m}, 2 \mathrm{H}), 1.99(\mathrm{~s}, 3 \mathrm{H}), 1.96(\mathrm{~m}, 2 \mathrm{H}), 1.85-1.75(\mathrm{~m}, 3 \mathrm{H}), 1.64-1.40$ $(\mathrm{m}, 7 \mathrm{H}), 1.31(\mathrm{~m}, 4 \mathrm{H}), 1.22(\mathrm{~m}, 1 \mathrm{H}), 1.15-1.03(\mathrm{~m}, 6 \mathrm{H}$ containing a doublet of $3 \mathrm{H}, J=7.3$ $\mathrm{Hz}), 1.03-0.87(\mathrm{~m}, 8 \mathrm{H}$ containing a singlet of $3 \mathrm{H}), 0.86(\mathrm{~d}, 3 \mathrm{H}, J=6.4 \mathrm{~Hz}), 0.63(\mathrm{~s}, 3 \mathrm{H}) ;{ }^{13} \mathrm{C}$ NMR $\left(125 \mathrm{MHz}, \mathrm{CDCl}_{3}\right) \delta 177.5,170.6,139.8,122.8,74.1,56.8,56.2,51.6,50.2,42.5,39.9$, $39.7,38.3,37.1,36.7,35.9,35.8,34.5,32.1,32.0,28.4,27.9,24.4,24.0,21.6,21.2,19.5$, 18.8, 17.4, 12.0; IR (film, $\left.\mathrm{cm}^{-1}\right)$ 2937, 1728, 1456, 1373, 1246; MS (ES +) m/z (\%): 473.7 (M $\left.+\mathrm{H}^{+}, 20\right)$. 


\section{Enone (7a)}
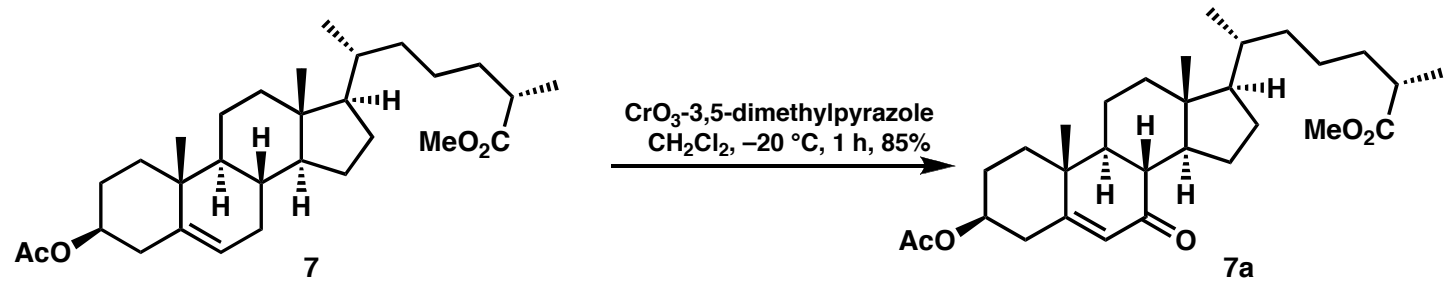

To a stirred solution of $\mathrm{CrO}_{3}(2.16 \mathrm{~g}, 21.7 \mathrm{mmol})$ in $\mathrm{CH}_{2} \mathrm{Cl}_{2}(22 \mathrm{~mL})$ was rapidly added 3,5-dimethylpyrazole $(2.08 \mathrm{~g}, 21.7 \mathrm{mmol})$ at $-20{ }^{\circ} \mathrm{C}$. The resulting reddish solution was stirred at $-20{ }^{\circ} \mathrm{C}$ for $20 \mathrm{~min}$ and $7(570 \mathrm{mg}, 1.2 \mathrm{mmol})$ was directly added in one portion. The solution was stirred for $1 \mathrm{~h}$ while allowing to warm to $-15{ }^{\circ} \mathrm{C}$. The solution was filtered through a pad of Celite ${ }^{\circledR}$ and evaporated to dryness. The residue was purified by flash chromatography (hexanes/EtOAc; 8:2) to give the $\Delta^{5}$-7-enone $7 \mathbf{a}(498 \mathrm{mg}, 85 \%)$ as a white solid. $[\alpha]_{\mathrm{D}}-90.0\left(c\right.$ 0.1, $\mathrm{CHCl}_{3}$ ); m.p. $108-110{ }^{\circ} \mathrm{C} ;{ }^{1} \mathrm{H}$ NMR $\left(400 \mathrm{MHz}, \mathrm{CDCl}_{3}\right) \delta 5.67$ (s, $1 \mathrm{H}) ; 4.68(\mathrm{~m}, 1 \mathrm{H}), 3.64(\mathrm{~s}, 3 \mathrm{H}), 2.53(\mathrm{~m}, 1 \mathrm{H}), 2.50-2.34(\mathrm{~m}, 3 \mathrm{H}), 2.20(\mathrm{t}, 1 \mathrm{H}, J=11.2 \mathrm{~Hz})$, $2.02(\mathrm{~s}, 3 \mathrm{H}), 1.95(\mathrm{~m}, 3 \mathrm{H}), 1.86(\mathrm{~m}, 1 \mathrm{H}), 1.65(\mathrm{~m}, 2 \mathrm{H}), 1.54(\mathrm{~m}, 4 \mathrm{H}), 1.40-1.18(\mathrm{~m}, 12 \mathrm{H}$ containing a singlet of $3 \mathrm{H}), 1.12(\mathrm{~d}, 3 \mathrm{H}, J=6.8 \mathrm{~Hz}), 1.04(\mathrm{~m}, 2 \mathrm{H}), 0.88(\mathrm{~d}, 3 \mathrm{H}, J=6.4 \mathrm{~Hz})$, 0.65 (s, 3H); ${ }^{13} \mathrm{C}$ NMR (125 MHz, $\left.\mathrm{CDCl}_{3}\right) \delta 201.9,177.4,170.3,164.0,126.2,72.3,54.8$, $51.6,50.1,49.9,45.5,43.2,39.6,38.8,38.4,37.9,36.1,35.9,35.7,34.4,28.7,27.5,26.4$, 23.9, 21.4, 21.3, 18.9, 17.4, 12.1; IR (film, $\mathrm{cm}^{-1}$ ) 2949, 1728, 1669, 1457, 1243, 1181, 1034; HRMS calcd for $\mathrm{C}_{30} \mathrm{H}_{47} \mathrm{O}_{5}\left(\mathrm{M}+\mathrm{H}^{+}\right)$487. 3423 found: 487. 3413.

Ketone (8)
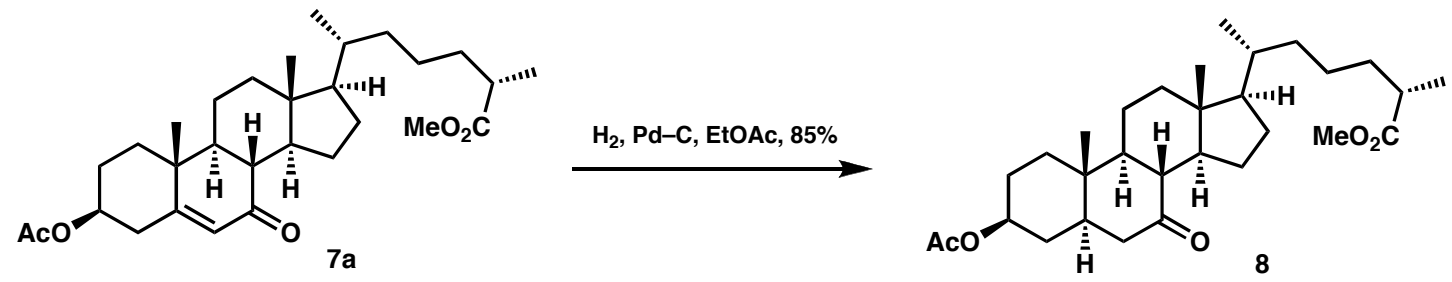
To a stirred solution of 7a $(1.16 \mathrm{~g}, 2.38 \mathrm{mmol})$ in THF $(24 \mathrm{~mL})$ was added $\mathrm{Pd} / \mathrm{C}(58 \mathrm{mg}, 5 \%$ w/w). A hydrogen-filled balloon (1 atm) was placed over the solution and the mixture was stirred for $3 \mathrm{~h}$ by which time TLC analysis indicated that the reaction was completed. The mixture was filtered through a pad of Celite ${ }^{\circledR}$ and washed with EtOAc (10 mL). After evaporation, the residue was purified by flash chromatography (hexanes/EtOAc; 8:2) to give $8(990 \mathrm{mg}, 85 \%)$ as a white solid. $[\alpha]_{\mathrm{D}}-4.5\left(\mathrm{c} 0.22, \mathrm{CHCl}_{3}\right)$; m.p. $122{ }^{\circ} \mathrm{C} ;{ }^{1} \mathrm{H} \mathrm{NMR}(400 \mathrm{MHz}$, $\left.\mathrm{CDCl}_{3}\right) \delta 4.60(\mathrm{~m}, 1 \mathrm{H}), 3.59(\mathrm{~s}, 3 \mathrm{H}), 2.36(\mathrm{q}, 1 \mathrm{H}, J=6.8 \mathrm{~Hz}), 2.27(\mathrm{t}, 2 \mathrm{H}, J=12.2 \mathrm{~Hz}), 2.13$ (m, 1H), $1.95(\mathrm{~s}, 3 \mathrm{H}), 1.90(\mathrm{~m}, 2 \mathrm{H}), 1.81(\mathrm{~m}, 2 \mathrm{H}), 1.73(\mathrm{~m}, 1 \mathrm{H}), 1.60(\mathrm{~m}, 2 \mathrm{H}), 1.52-1.38(\mathrm{~m}$, 5H), 1.38-1.21 (m, 6H), $1.18(\mathrm{~m}, 2 \mathrm{H}), 1.06(\mathrm{~d}, 3 \mathrm{H}, J=7.3 \mathrm{~Hz}), 1.03(\mathrm{~s}, 3 \mathrm{H}), 1.00(\mathrm{~m}, 4 \mathrm{H})$, $0.85(\mathrm{~m}, 1 \mathrm{H}), 0.82(\mathrm{~d}, 3 \mathrm{H}, J=6.4 \mathrm{~Hz}), 0.57(\mathrm{~s}, 3 \mathrm{H}) ;{ }^{13} \mathrm{C} \mathrm{NMR}\left(125 \mathrm{MHz}, \mathrm{CDCl}_{3}\right) \delta 211.7$, $177.4,170.5,72.9,55.1(2), 51.5,50.0,49.0,46.6,46.0,42.6,39.6,38.8,36.1,36.0,35.9$, 35.6, 34.4, 34.0, 28.5, 27.2, 25.1, 23.9, 21.9, 21.5, 18.8, 17.4, 12.2, 11.8; IR (film, cm ${ }^{-1}$ ) 2942, 1733, 1705, 1558, 1456, 1239, 1024; MS (ES +) $m / z(\%): 489.7\left(\mathrm{M}+\mathrm{H}^{+}, 40\right)$.

\section{$\Delta^{7}$-Unsaturated Ester (9)}
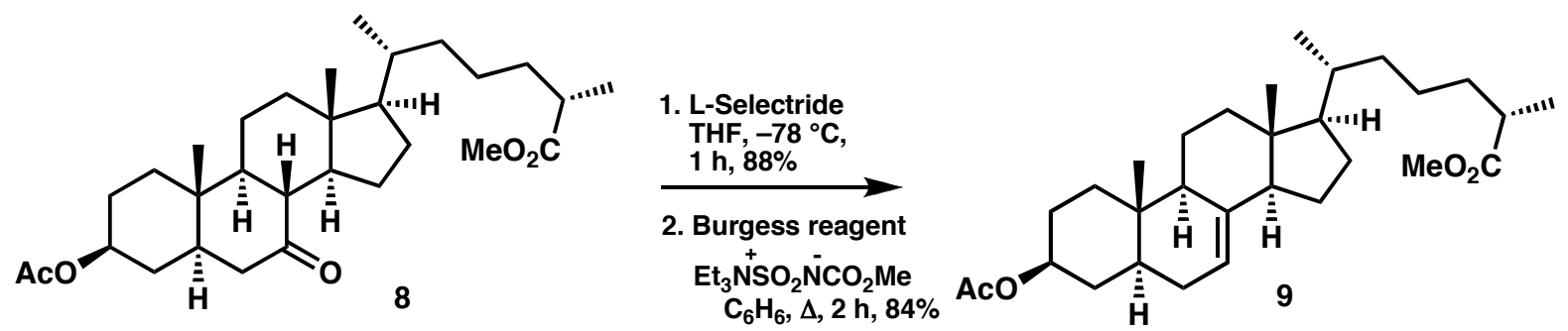

To a stirred solution of 8 (990 $\mathrm{mg}, 0.116 \mathrm{mmol})$ in THF $(20 \mathrm{~mL})$ was slowly added L-Selectride ${ }^{\circledR}\left(2.62 \mathrm{~mL}, 2.62 \mathrm{mmol}, 1 \mathrm{M}\right.$ in THF) at $-78^{\circ} \mathrm{C}$. The resulting solution was stirred at that temperature for $1 \mathrm{~h}$ and quenched with sat. $\mathrm{NaHCO}_{3}(\mathrm{aq})(10 \mathrm{~mL}), \mathrm{H}_{2} \mathrm{O}_{2}(50 \%$ aq. $)(10$ $\mathrm{mL})$ and diluted with EtOAc $(20 \mathrm{~mL})$. The solution was stirred at r.t. for $1 \mathrm{~h}$ and the aqueous phase was extracted with EtOAc $(3 \times 20 \mathrm{~mL})$. The combined organic phases were dried with $\mathrm{Na}_{2} \mathrm{SO}_{4}$ and evaporated to dryness. The residue was purified by flash chromatography 
(hexanes/EtOAc; $7: 3$ ) to give the $7-\alpha-\mathrm{OH}$ compound as a single diastereomer (878 $\mathrm{mg}, 88 \%$ ) that was directly submitted to the next step. To a stirred solution of the above alcohol $(52 \mathrm{mg}$, $0.105 \mathrm{mmol})$ in $\mathrm{C}_{6} \mathrm{H}_{6}(1 \mathrm{~mL})$ was added Burgess reagent $\left(\mathrm{Et}_{3} \mathrm{~N}^{+} \mathrm{SO}_{2} \mathrm{~N}^{-} \mathrm{CO}_{2} \mathrm{Me}\right)(50 \mathrm{mg}, 0.211$ mmol) at room temperature. The solution was then heated at reflux temperature for $2 \mathrm{~h}$ by which time TLC analysis showed complete conversion. The solution was directly submitted to flash chromatography (hexanes/EtOAc; 9:1) to give $9(41 \mathrm{mg}, 84 \%)$ as a white solid. $[\alpha]_{\mathrm{D}}$ $+16.0\left(c\right.$ 0.05, $\left.\mathrm{CHCl}_{3}\right)$; m.p. $95{ }^{\circ} \mathrm{C} ;{ }^{1} \mathrm{H} \mathrm{NMR}\left(500 \mathrm{MHz}, \mathrm{CDCl}_{3}\right) \delta 5.12(\mathrm{~s}, 1 \mathrm{H}), 4.67(\mathrm{~m}, 1 \mathrm{H})$, $3.65(\mathrm{~s}, 3 \mathrm{H}), 2.41(\mathrm{q}, 1 \mathrm{H}, J=6.3 \mathrm{~Hz}), 2.00(\mathrm{~s}, 3 \mathrm{H}), 1.98(\mathrm{~m}, 1 \mathrm{H}), 1.86-1.83(\mathrm{~m}, 4 \mathrm{H}), 1.81(\mathrm{~m}$, 2H), 1.80-1.60 (m, 4H), 1.60-1.40 (m, 4H), $1.34(\mathrm{~m}, 6 \mathrm{H}), 1.11(\mathrm{~m}, 2 \mathrm{H}), 1.12(\mathrm{~d}, 3 \mathrm{H}, J=6.8$ $\mathrm{Hz})+(\mathrm{m}, 2 \mathrm{H}), 1.01(\mathrm{~m}, 1 \mathrm{H}), 0.89(\mathrm{~d}, 3 \mathrm{H}, J=6.8 \mathrm{~Hz}), 0.79(\mathrm{~s}, 3 \mathrm{H}), 0.50(\mathrm{~s}, 3 \mathrm{H}) ;{ }^{13} \mathrm{C} \mathrm{NMR}$ $\left(125 \mathrm{MHz}, \mathrm{CDCl}_{3}\right) \delta 177.6,170.9,139.7,117.5,73.6,56.2,55.1,51.6,49.4,43.5,40.2$, $39.7(2), 37.0,36.2,35.9,34.5,34.4,34.0,29.7,28.9,28.1,27.7,24.0,23.1,21.6,18.9,17.4$, 13.1, 12.0; IR (film, $\mathrm{cm}^{-1}$ ) 2949, 1718, 1558, 1249, 1215, 1030; HRMS calcd for $\mathrm{C}_{30} \mathrm{H}_{49} \mathrm{O}_{4}(\mathrm{M}$ $\left.+\mathrm{H}^{+}\right) 473.3631$ found: 473.3635 .

\section{Dafachronic acid A (1)}

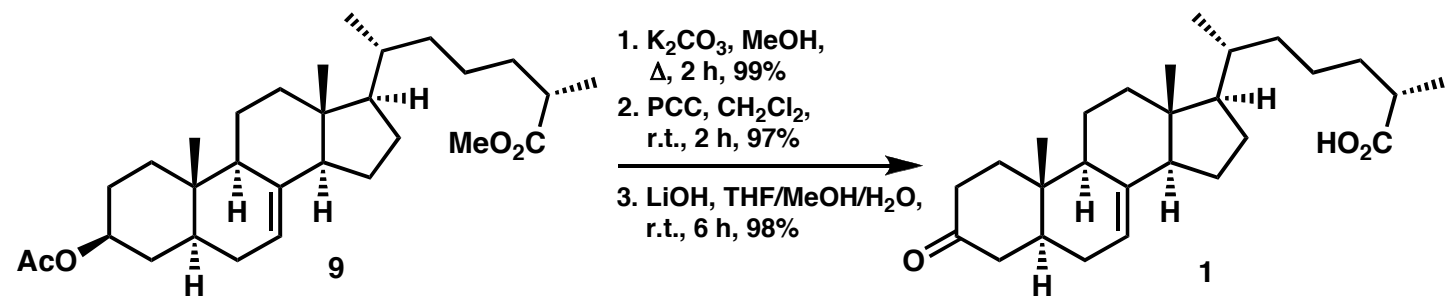

To a stirred solution of 3- $\beta$-acetate $9(574 \mathrm{mg}, 0.198 \mathrm{mmol})$ in $\mathrm{MeOH}(12 \mathrm{~mL})$ was added $\mathrm{K}_{2} \mathrm{CO}_{3}(84 \mathrm{mg}, 0.607 \mathrm{mmol})$ and the solution was refluxed for $2 \mathrm{~h}$. The solution was cooled to r.t. and acidified with $1 N \mathrm{HCl}(4 \mathrm{~mL})$. The aqueous phase was extracted with EtOAc $(3 \mathrm{x} 10$ $\mathrm{mL}$ ) and the combined organic phase were dried over $\mathrm{Na}_{2} \mathrm{SO}_{4}$ and evaporated to dryness. The residue was purified by flash chromatography (hexanes/EtOAc; 7:3) to give the deacetylated 
3- $\beta$-alcohol (514 mg, 99\%), which was submitted directly to the oxidation step. To a stirred solution of the $3-\beta$-alcohol $(514 \mathrm{mg}, 1.19 \mathrm{mmol})$ in $\mathrm{CH}_{2} \mathrm{Cl}_{2}(15 \mathrm{~mL})$ was added pyridinium chlorochromate (PCC) $(334 \mathrm{mg}, 1.55 \mathrm{mmol})$ at r.t. The solution was stirred at that temperature for $2 \mathrm{~h}$ and filtered through a short pad of Celite ${ }^{\circledR}$ which was washed with $\mathrm{CH}_{2} \mathrm{Cl}_{2}$. After evaporation of the filtrate, the residue was purified by flash chromatography (hexanes/EtOAc; 8:2) to give the corresponding 3-ketoester (490 mg, 97\%) which was submitted directly to the hydrolysis step. To a stirred solution of the above ester $(490 \mathrm{mg}$, $1.14 \mathrm{mmol})$ in $\mathrm{THF} / \mathrm{MeOH} / \mathrm{H}_{2} \mathrm{O}(2: 1: 1)(11 \mathrm{~mL})$ was added $\mathrm{LiOH} \cdot \mathrm{H}_{2} \mathrm{O}(956 \mathrm{mg}, 3.48 \mathrm{mmol})$ at r.t. and the reaction was stirred for $6 \mathrm{~h}$ by which time TLC analysis showed complete conversion. The reaction mixture was acidified by $3 M \mathrm{HCl}$ and the aqueous phase was extracted with EtOAc $(3 \times 25 \mathrm{~mL})$. The combined organic phase were dried over $\mathrm{Na}_{2} \mathrm{SO}_{4}$ and evaporated to dryness. The residue was purified by flash chromatography (hexanes/EtOAc; 7:3) to give $1(466 \mathrm{mg}, 98 \%)$ as a white solid. $[\alpha]_{\mathrm{D}}+23.4\left(c\right.$ 2.0, $\left.\mathrm{CDCl}_{3}\right)$; m.p. $143{ }^{\circ} \mathrm{C}{ }^{1} \mathrm{H}$ $\operatorname{NMR}\left(500 \mathrm{MHz}, \mathrm{CDCl}_{3}\right) \delta 5.16(\mathrm{~s}, 1 \mathrm{H}), 2.45(\mathrm{~m}, 1 \mathrm{H}), 2.40(\mathrm{dd}, 1 \mathrm{H}, J=5.8$ and $14.6 \mathrm{~Hz})$, $2.25(\mathrm{~m}, 3 \mathrm{H}), 2.11(\mathrm{~m}, 1 \mathrm{H}), 2.05(\mathrm{~m}, 1 \mathrm{H}), 1.81(\mathrm{~m}, 5 \mathrm{H}), 1.78-1.58(\mathrm{~m}, 4 \mathrm{H}), 1.52(\mathrm{~m}, 3 \mathrm{H})$, $1.48-1.32(\mathrm{~m}, 7 \mathrm{H}), 1.30-1.18(\mathrm{~m}, 5 \mathrm{H}), 1.16(\mathrm{~d}, 3 \mathrm{H}, J=6.8 \mathrm{~Hz}), 1.01(\mathrm{~m}, 1 \mathrm{H}), 0.90(\mathrm{~d}, 3 \mathrm{H}, J=$ $6.8 \mathrm{~Hz}), 0.54(\mathrm{~s}, 3 \mathrm{H}) ;{ }^{13} \mathrm{C} \mathrm{NMR}\left(125 \mathrm{MHz}, \mathrm{CDCl}_{3}\right)$ 212.3, 183.3, 139.7, 117.2, 56.2, 55.1, 49.0, 44.4, 43.5, 43.0, 39.6(2), 38.9, 38.3, 36.2, 35.9, 34.6, 34.2, 30.2, 28.1, 24.0, 23.1, 21.9, 18.9, 17.2, 12.6. 12.1; IR (film, $\mathrm{cm}^{-1}$ ) 2939, 2869, 1733, 1701, 1684, 1653, 1558, 1457, 1215; HRMS calcd for $\mathrm{C}_{27} \mathrm{H}_{43} \mathrm{O}_{3}\left(\mathrm{M}+\mathrm{H}^{+}\right) 415.3206$ found: 415.3211 .

\section{References.}

(1) The phosphonate $\mathbf{3}$ was prepared according to a literature procedure: Kitahara, T.; Horiguchi, A.; Mori, K. Tetrahedron 1988, 44, 4713.

(2) Aldehyde 2 was prepared in large amounts according to literature procedures in: (a) Steele, J. A.; Mosettig, E. J. Org. Chem. 1963, 28, 571-572. (b) Salmond, W. G.; Sobala, M. C. Tetrahedron Lett. 1977, 18, $1695-1698$. 
STANDARD PROTON PARAMETERS

exp1 s2pul

SAMPLE
date Feb 192007 dfrq $\&$ VT
500.176 \begin{tabular}{rrrr} 
solvent & CDCl3 & dn & exp \\
file & dpwr & H1 \\
\hline
\end{tabular}

$\begin{array}{lrlr}\text { sfrq } & 500.176 & \mathrm{dm} & 0 \\ \mathrm{~m} & \mathrm{nnn}\end{array}$

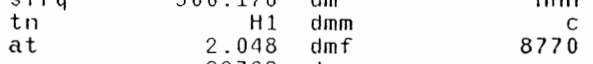

$\begin{array}{rrrr}\text { at } & 2.048 & \text { dmf } & \\ \text { np } & 32768 & \text { dseq } & \\ \text { sw } & 8000.0 & \text { dres } & 1.0 \\ \text { fb } & 4000 & \text { homo } & n\end{array}$

$\begin{array}{rrrr}\mathrm{fb} & 4000 & \text { homo } & n \\ \mathrm{bS} & 8 & \text { temp } & \text { n } \\ & 22.0\end{array}$

\begin{tabular}{lrll} 
SS & 2 & \multicolumn{1}{c}{ PROCESSING } \\
tpwr & 58 & ib & 0.10
\end{tabular}

$\begin{array}{lrll}\text { pw } & 5.0 & \text { wtfile } & \\ d 1 & 0 & \text { proc } & f t \\ \text { tof } & 0 & \text { fn } & \text { not used }\end{array}$

nt 10000 math not used

$\begin{array}{lrl}c t & 64 & \\ \text { a lock } & \text { n werr }\end{array}$

gain not used $\begin{array}{ll}\text { wexp } \\ \text { wbs }\end{array}$

i) 1 in wht

$\begin{array}{ll}\text { dp } & \text { y } \\ \text { hs } & \text { nn }\end{array}$

DISPLAY

$\begin{array}{rr}\mathrm{sp} & 48.3 \\ w p & 4897.9 \\ v s & 127\end{array}$

$\begin{array}{lr}\text { VS } & 127 \\ \text { SC } & 0 \\ \text { WC } & 250 \\ \text { hzmm } & 19.59 \\ \text { rf } & 536.08\end{array}$

$\begin{array}{lr}\text { hzmm } & 19.59 \\ \text { is } & 536.08 \\ \text { if } & 1499.0\end{array}$

$\begin{array}{rr}\mathrm{rfp} & 1499.0 \\ \mathrm{th} & 7\end{array}$

ins cad ph

wft
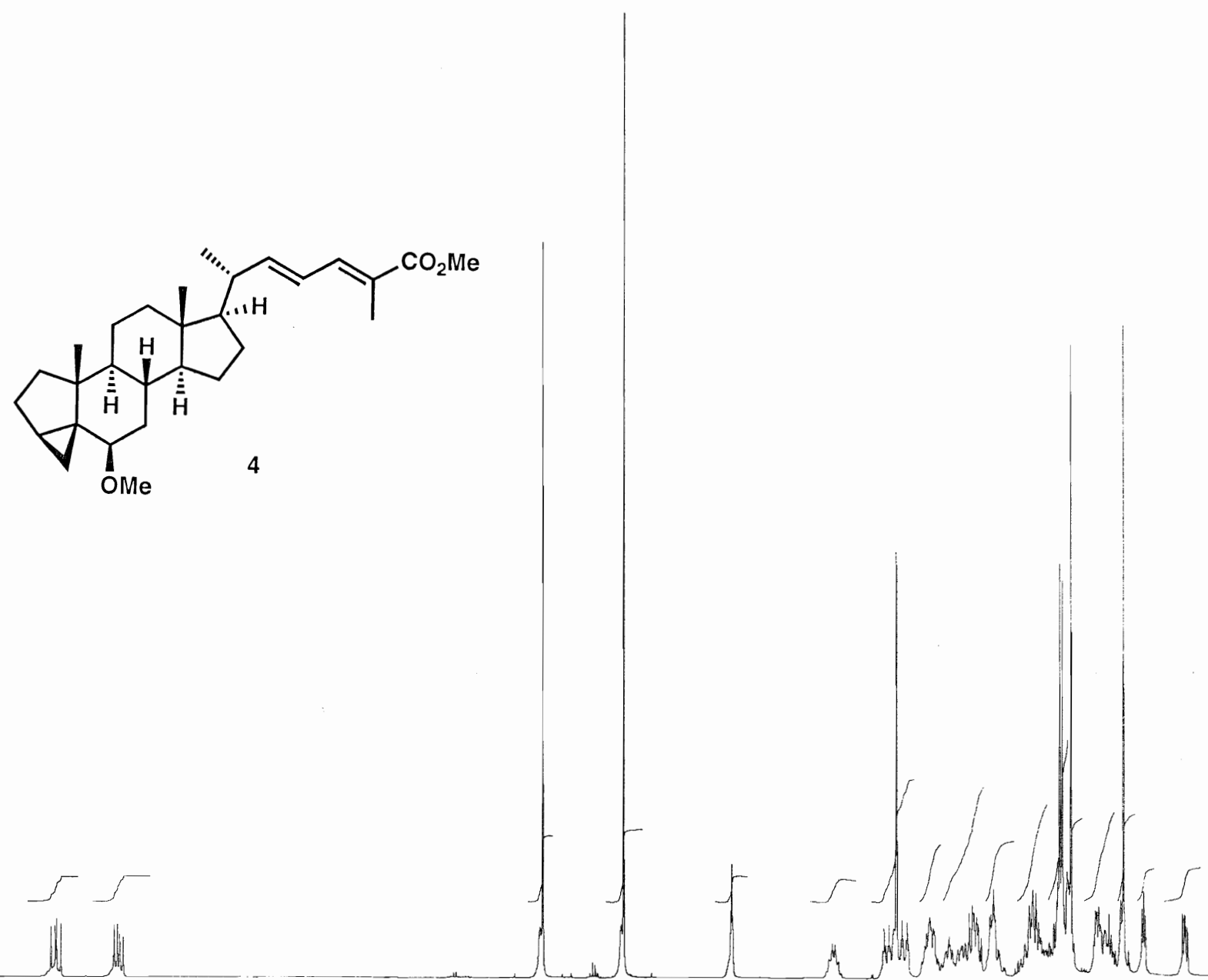

9

8

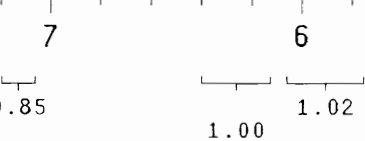

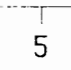

4

$2.67 \quad 2.90 \quad 1.01$

$0.83 \quad 2.332 .456 .813 .651 .33$
4.94
$4.63 \quad 4.003 .383 .541 .36$ 
13C OBSERVE
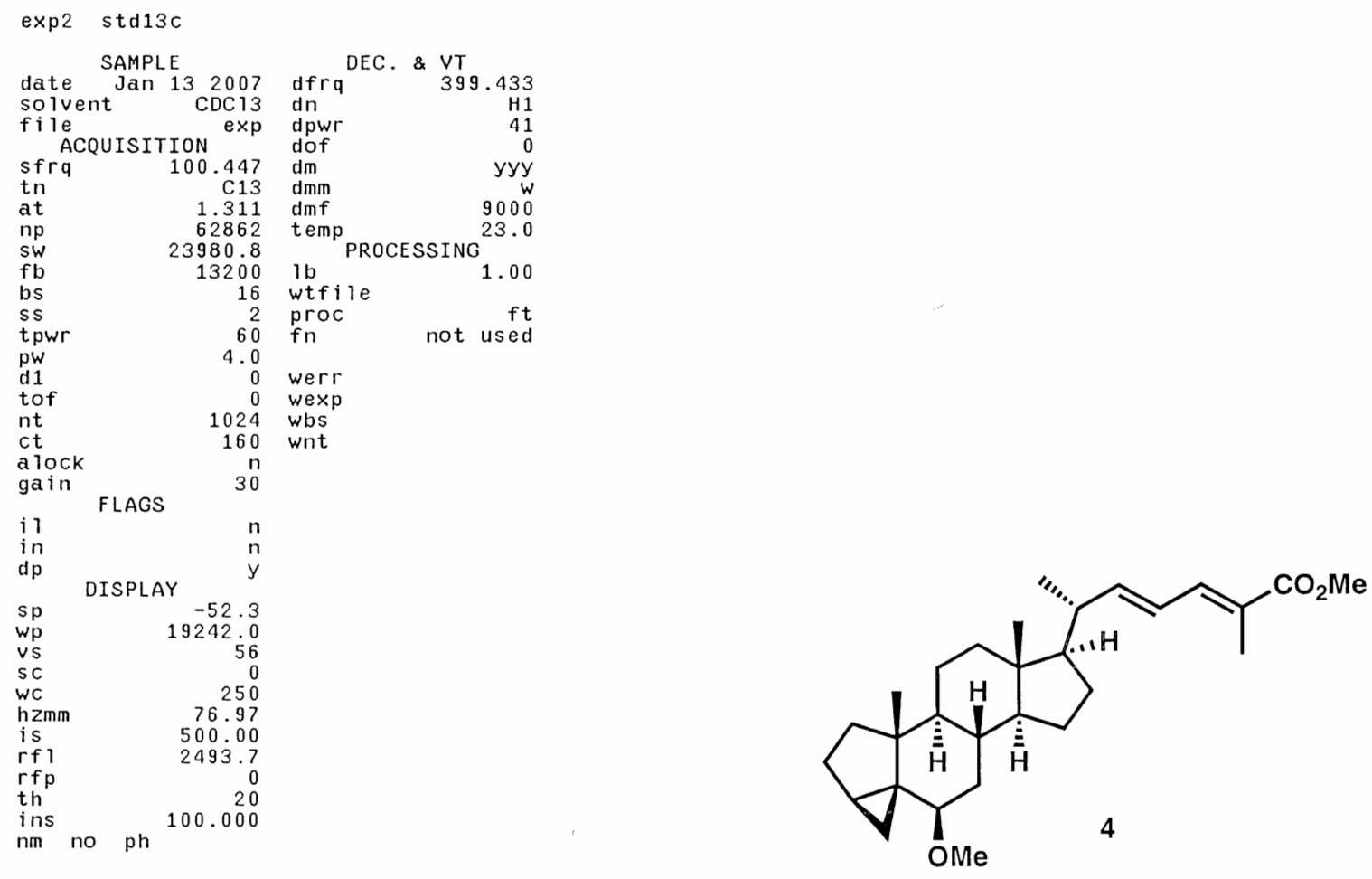

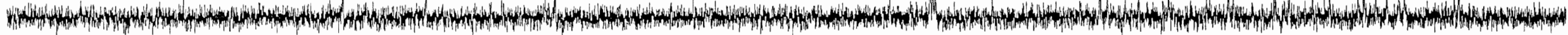

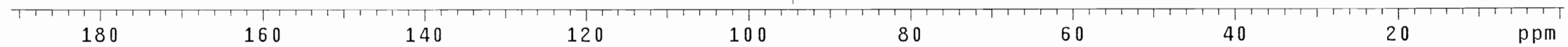


STANDARD 111 OBSERVE

exp1 std1h

SAMPLE
date Jan 25 2007 dffr DEC. \& VT
solvent

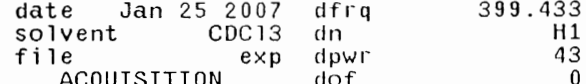
$\begin{array}{llll}\text { sfrq } & 399.433 & \mathrm{dm} & \mathrm{m} \\ \mathrm{tn} & 2.731 & \mathrm{dm}\end{array}$

$\begin{array}{lrlr}\mathrm{tn} & \mathrm{H} 1 & \mathrm{dmm} & \mathrm{C} \\ \mathrm{at} & 2.731 & \mathrm{dmf} & 12200\end{array}$

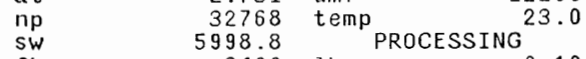

$\begin{array}{lrl}\mathrm{SW} & 5998.8 & \mathrm{lb} \text { PROCESSING } \\ \mathrm{fb} & 3400.10 & \mathrm{~b}\end{array}$

$\begin{array}{lrlr}\text { bs } & 8 & \text { wtfile } & \\ \text { ss } & 2 & \text { proc } & \text { nt } \\ \text { tpwr } & 59 & \text { fn } & \text { not used }\end{array}$

$\begin{array}{lrl}\mathrm{tpwr} & 59 & \mathrm{fn} \\ \mathrm{pw} & 12.0 & \end{array}$

$\begin{array}{lrl}\text { d1 } & 0 & \text { werr } \\ \text { tof } & 0 & \text { wexp } \\ \text { nt } & 100 & \text { whs }\end{array}$

$\begin{array}{lrl}\mathrm{nt} & 1000 & \text { wbs } \\ \mathrm{ct} & 88 & \text { wht }\end{array}$

alock not used
gain

il FLAGS

$\begin{array}{ll}\text { in } & n \\ d p & y\end{array}$

$\begin{array}{rr}\text { sp } & \text { DISPLAY } \\ \text { wp } & 54.6 \\ \text { sc } & 3110.9\end{array}$

$\begin{array}{lr}\text { wp } & 3110.9 \\ \text { vs } & 100\end{array}$

$\begin{array}{lr}\text { SC } & 0 \\ \text { WC } & 250 \\ \text { hZmm } & 12.44\end{array}$

$\begin{array}{ll}\text { is } & 820.86 \\ \mathrm{rfl} & 1031.8\end{array}$

rfp

$\begin{array}{lr}r f p & 20 \\ \text { th } & 20 \\ \text { ins } & 1.000\end{array}$
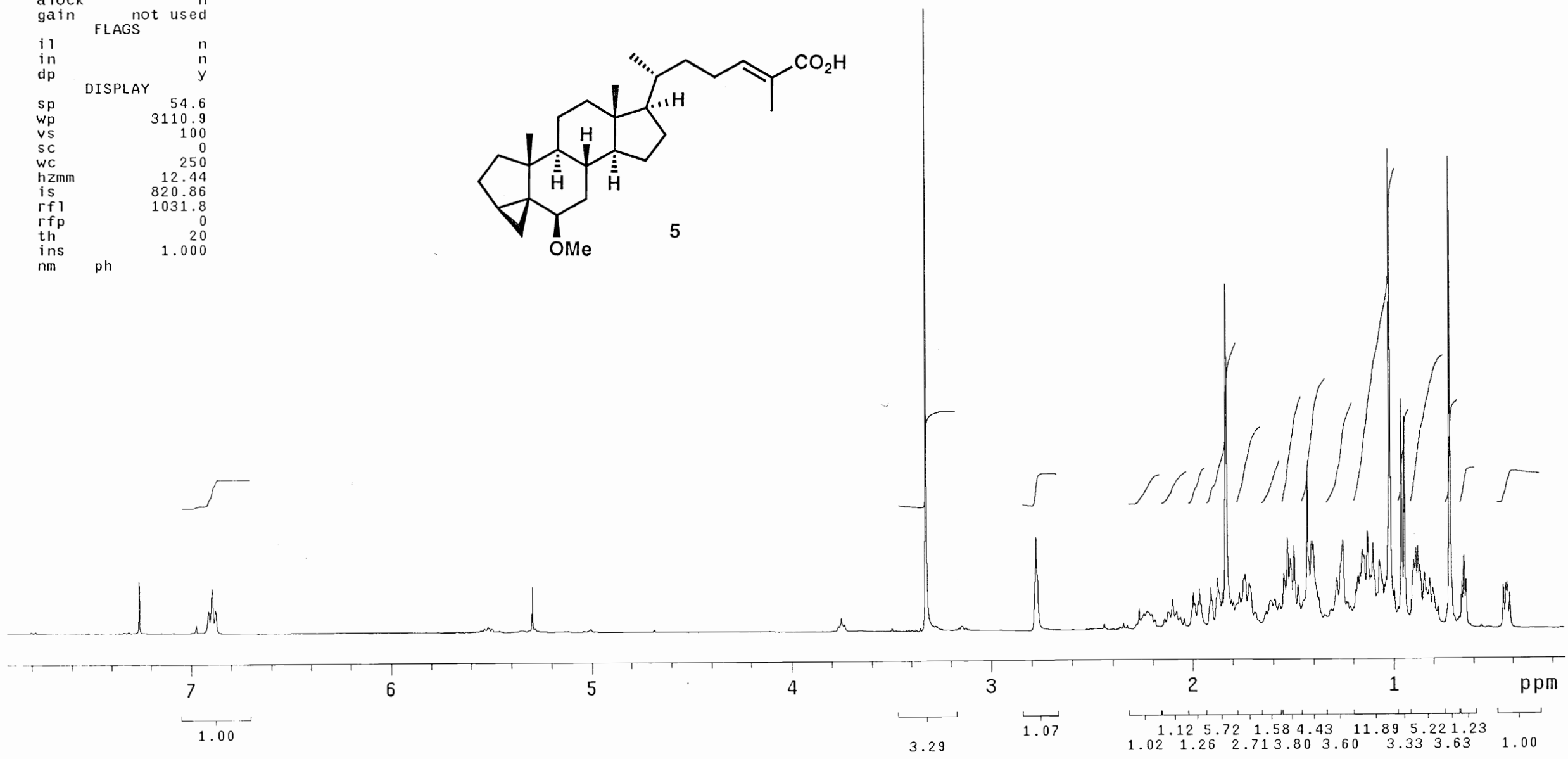
13C OBSERVE
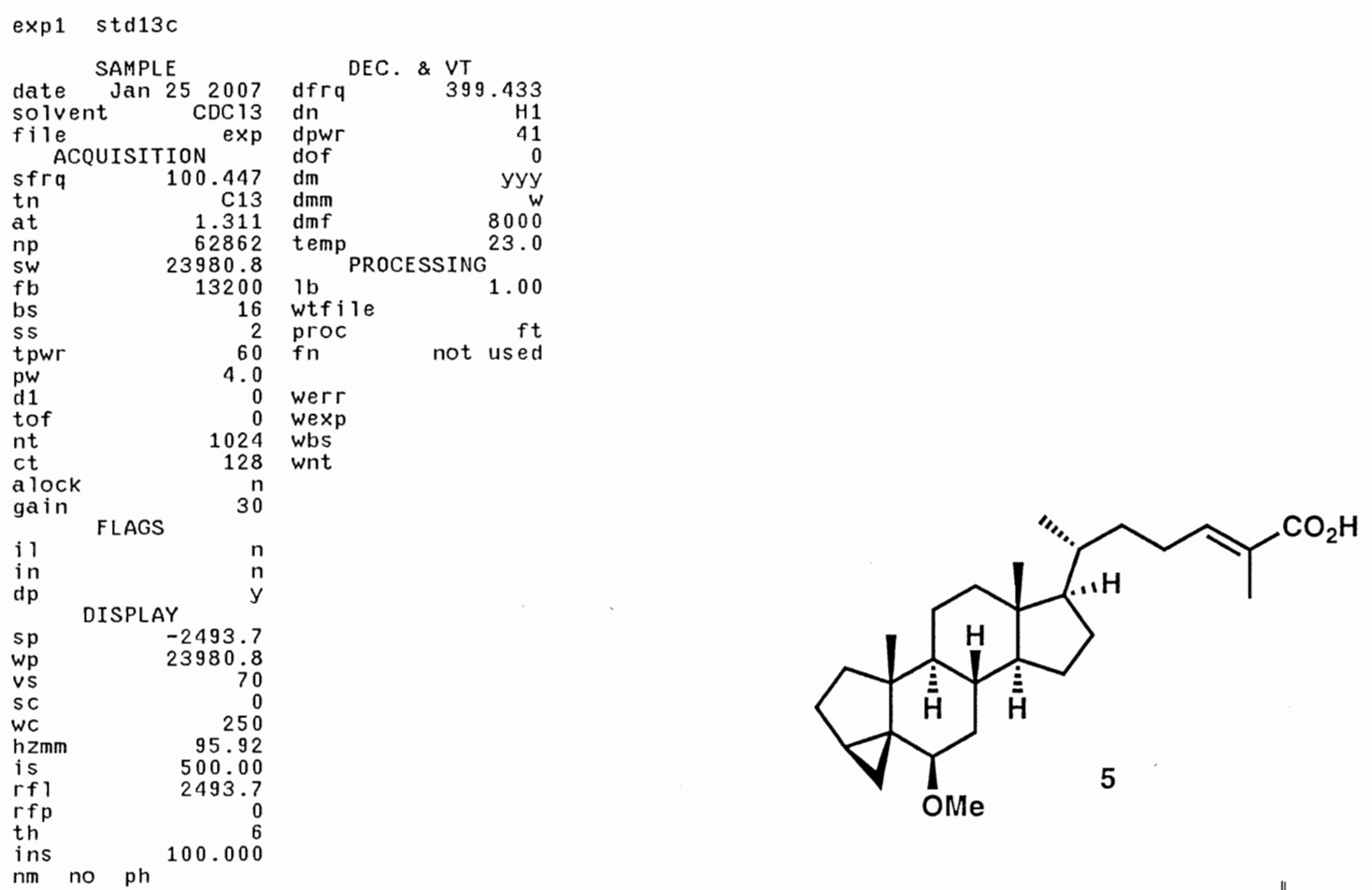

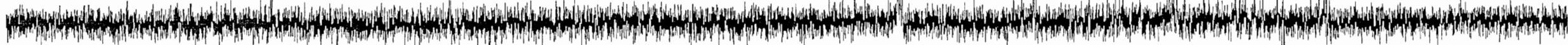


STANDARD PROTON PARAMETERS

exp2 s2pul

\begin{tabular}{llll}
\multicolumn{2}{c}{ SAMPLE } & \multicolumn{2}{c}{ DEC. \& VT } \\
date Mar 16 2007 & dfrq & 500.176 \\
solvent & CDC13 & dn &
\end{tabular}

$\begin{array}{crlr}\text { solvent } & \operatorname{CDCl3} & \text { dn } & \text { H1 } \\ \text { file } & \text { exp } & \text { dpwr } & 32 \\ \text { ACQUISITION } & \text { dof } & 0\end{array}$

$\begin{array}{lrlr}s f r q & 500.176 & d m & n \\ t n & H 1 & d m m & n \\ \text { t } & 2.048 & d m f & c \\ \text { t } & 32768 & d s e q & 8770\end{array}$

$\begin{array}{rrrr}\mathrm{np} & 32768 & \text { dseq } & \\ \mathrm{sw} & 8000.0 & \text { dres } & 1.0 \\ \mathrm{fb} & 4000 & \text { homo } & \text { n } \\ \text { bs } & 8 & \text { temp } & 22.0\end{array}$

$\begin{array}{lrl}\text { bs } & 8 & \text { temp } \\ \text { ss } & 2 & \text { PROCESSING }\end{array}$

tpwr $58 \mathrm{lb} \quad 0.10$

$\begin{array}{lrll}\mathrm{pw} & 5.0 & \text { wtfile } & \mathrm{ft} \\ \mathrm{d} 1 & 0 & \text { proc } & \mathrm{ft}\end{array}$

$\begin{array}{lrl}\text { tof } & 0 & \text { fn } \\ \text { nt } & 10000 & \text { math used }\end{array}$

a lock $n$ werr

gain not used wexp

il FLAGS $n$ wht

dp

$\begin{array}{rr}\text { sp } & -98.1 \\ \text { Wp } & 3810.5 \\ \text { vs } & 127\end{array}$

$\begin{array}{lr}\text { SC } & 0 \\ \text { WC } & 250 \\ \text { hZmm } & 15.24\end{array}$

$\begin{array}{lr}\text { hzmm } & 15.24 \\ \text { is } & 339.33 \\ r f 7 & 1499.0\end{array}$

$\begin{array}{rr}r f p & 1499.0 \\ r & 0\end{array}$

ins cdc ph

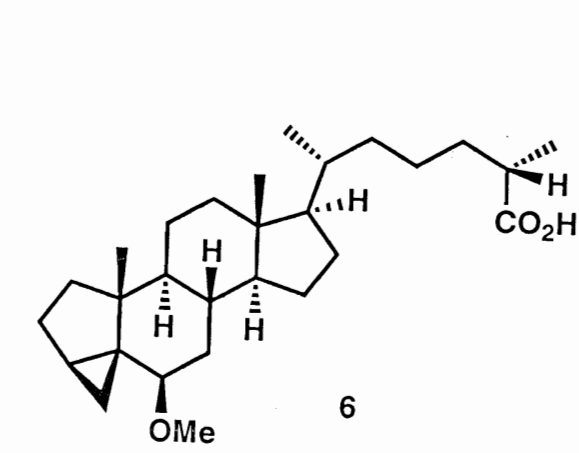

$w f t$

.

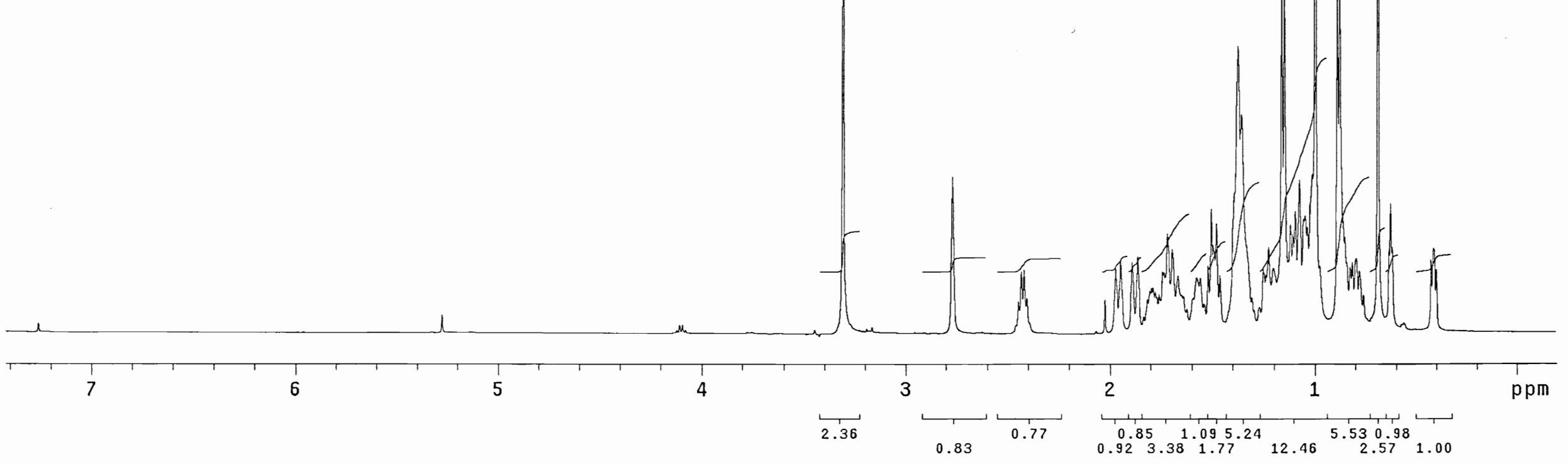


STANDARD CARBON PARAMETERS

exp1 s2put

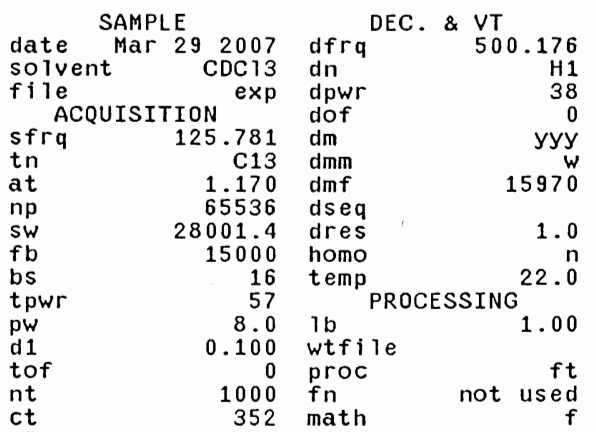

alock 352 math

gain 56 werr

$\begin{array}{lll}\text { in } & \text { FLAGS } & \text { wexp } \\ \text { in } & n & \text { wbs } \\ \text { in } & n & \text { wht }\end{array}$

dp
hs

$\begin{array}{lr}\text { sp } & 1153.4 \\ \text { wp } & 22544.3\end{array}$

wp $\quad 22544.3$

$\begin{array}{lr}\text { SC } & 0 \\ \text { WC } & 250 \\ \text { hZmm } & 90.18\end{array}$

$\begin{array}{lr}\text { hZmm } & 90.18 \\ \text { is } & 500.00 \\ \text { rfl } & 2051.1\end{array}$

$\begin{array}{lr}\mathrm{rfp} & 0 \\ \mathrm{th} & 68\end{array}$

ins 100.000
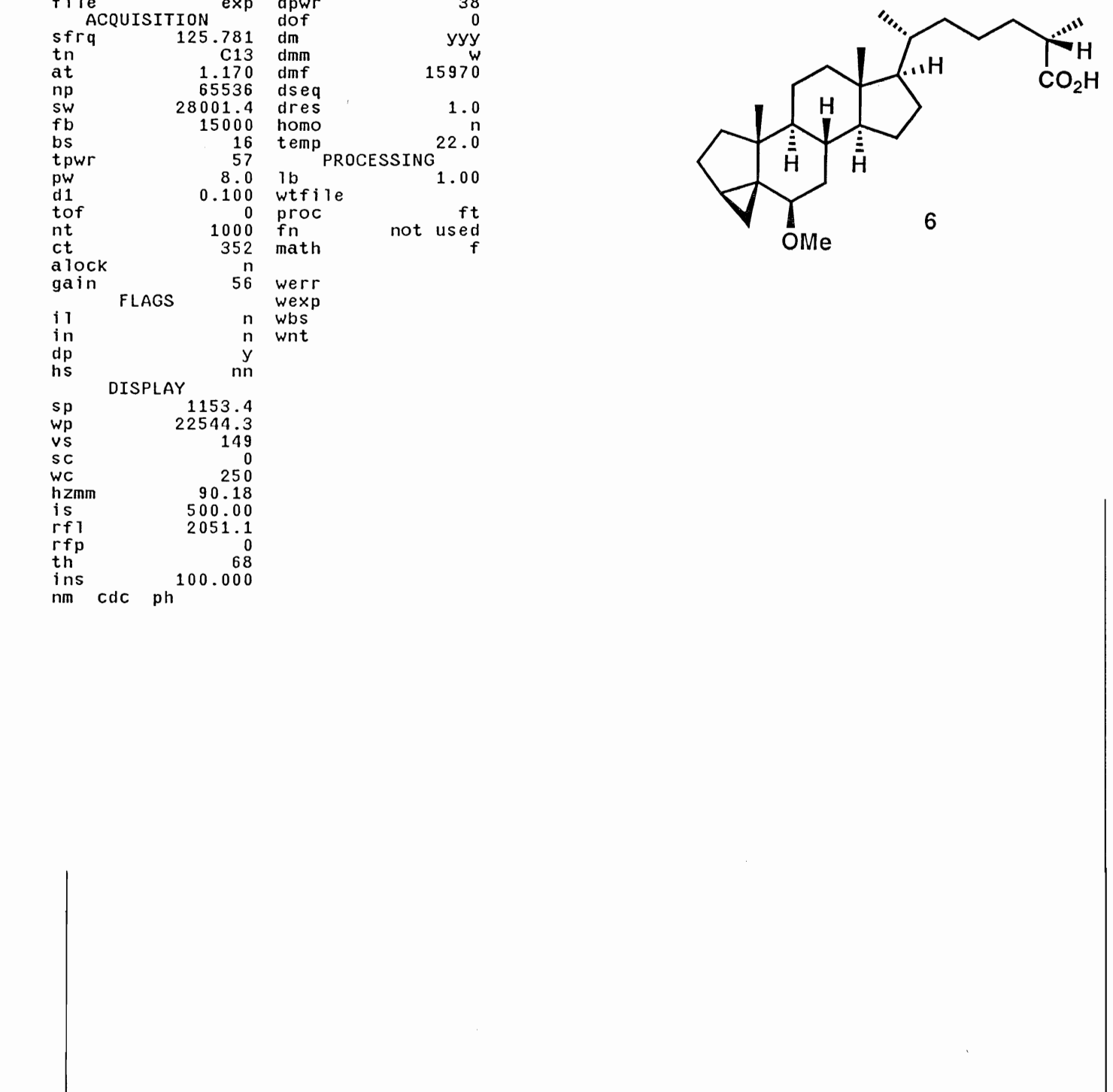

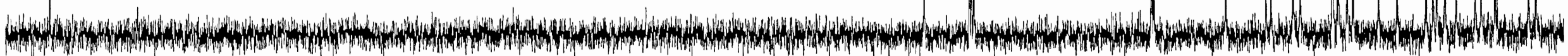

180

160

140

120

100

80

60

40

$20 \mathrm{ppm}$ 
STANDARD PROTON PARAMETERS

exp1 s2pul

$\begin{array}{llll}\text { SAMPLE } & \text { DEC. \& VT } \\ \text { date Feb } 182007 & \text { dfrq } & 500.176 \\ \text { solvent } & \text { CDC13 } & \text { dn } & \text { H1 }\end{array}$

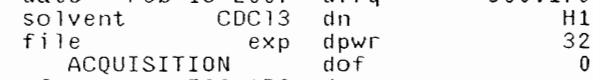

$\begin{array}{cccc}\text { ACQUISITION } & \text { dof } & 0 \\ \text { sfrq } & 500.176 & \text { dm } & n n n\end{array}$

$\begin{array}{lrlr}\mathrm{tn} & \mathrm{H} 1 & \mathrm{dmm} & \mathrm{c} \\ \mathrm{at} & 2.048 & \mathrm{dmf} & 8770\end{array}$

$\begin{array}{rrrr}\mathrm{np} & 32768 & \mathrm{dseq} & \\ \mathrm{sw} & 8000.0 & \text { dres } & 1.0 \\ \mathrm{fb} & 4000 & \text { homo } & \mathrm{n}\end{array}$

$\begin{array}{rrrr}\text { fo } & 4000 & \text { homo } \\ \text { bs } & 8 & \text { temp } \\ \text { ss } & 2 & \text { PROCESSING }\end{array}$

tpwr 58 ib 0.10

$\begin{array}{lrlr}\text { pw } & 5.0 & \text { wtfile } & \\ \text { d1 } & 0 & \text { proc } \\ \text { tof } & 0 & \text { fn } & \text { not used }\end{array}$

$\begin{array}{lrl}\text { tof } & 0 & \text { fn } \\ \text { nt } & 1000 & \text { math } \\ c t & 64 & \end{array}$

$\begin{array}{lrl}\text { ct } & \text { n werr } \\ \text { alock } & \text { nat used } \\ \text { gain wexp }\end{array}$

gain not used wexp

$\begin{array}{lll}i & \text { FLAGS } & \text { wbs } \\ \text { in wht } & n \text { wft }\end{array}$

in $\mathrm{dp} \quad \mathrm{n}$

hs DISPLAY nn

$\begin{array}{rr}\mathrm{sp} & \text { DISPLAY } \\ \mathrm{wp} & 247.1 \\ \mathrm{vs} & 3397.0\end{array}$

$\begin{array}{lr}\text { wp } & 3397.0 \\ \text { VS } & 152 \\ \text { SC } & 0\end{array}$

$\begin{array}{lr}\text { WC } & 250 \\ \text { hzmm } & 13.59\end{array}$

$\begin{array}{lr}\text { hzmm } & 13.59 \\ \text { is } & 1456.31\end{array}$

$\begin{array}{lr}\text { rf } & 1499.0 \\ \text { ifp } & 0 \\ \text { th } & 7 \\ \text { ins } & 1.000\end{array}$

inm cdc ph

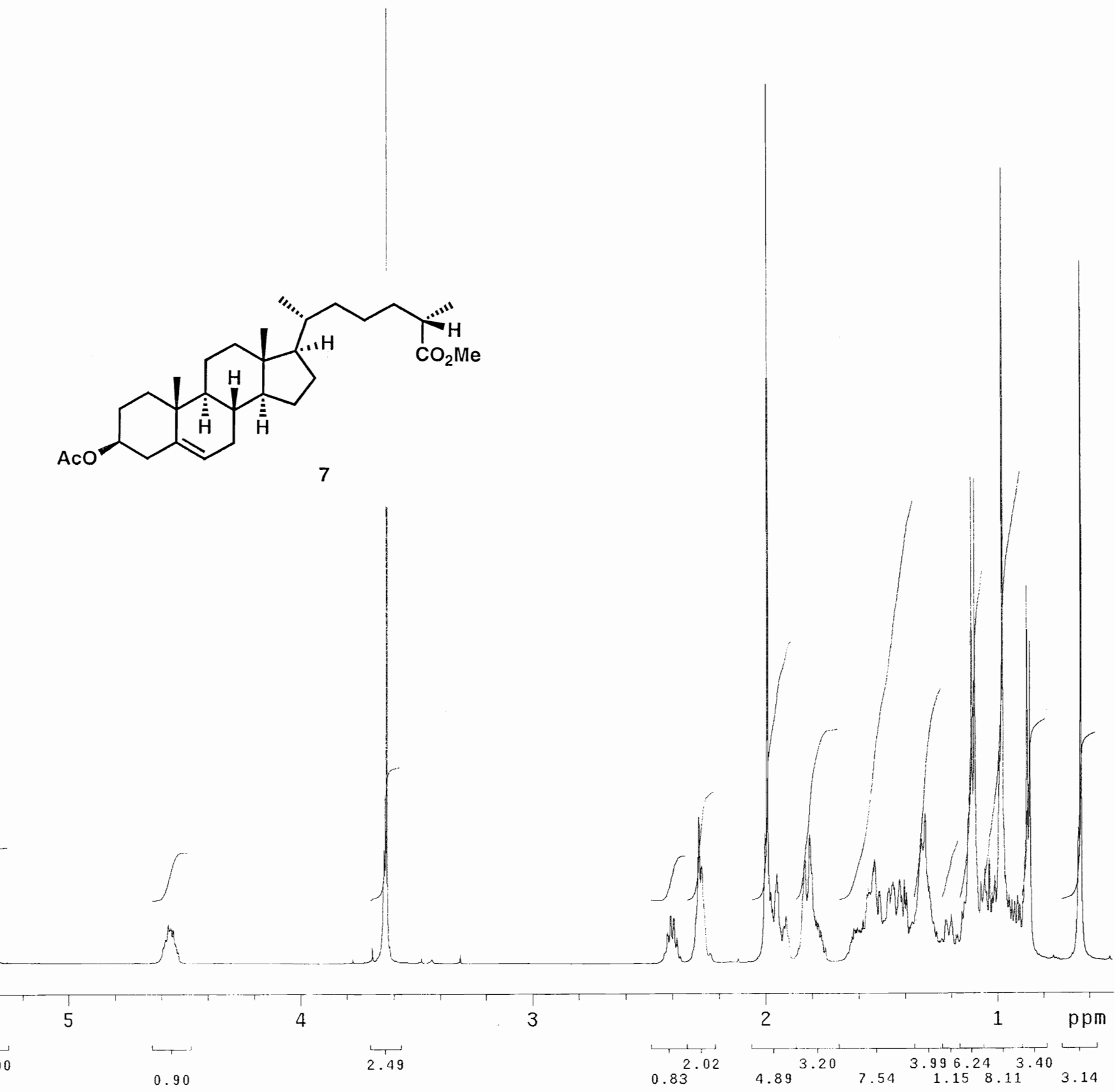

1.000 

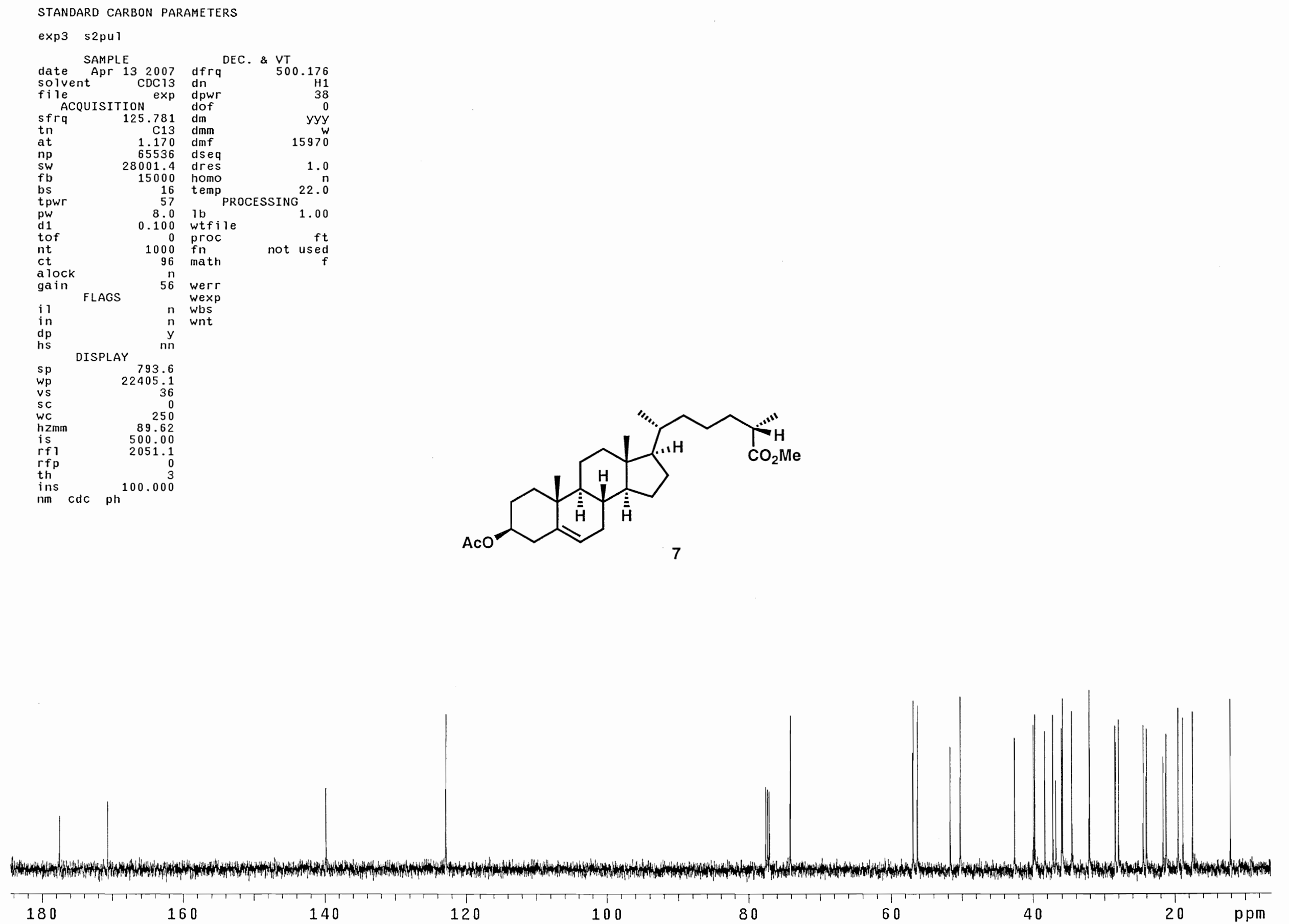
STANDARD PROTON PARAMETERS

exp1 s2pul

$\begin{array}{lll}\text { SAMPLE } & \text { DEC. \& VT } \\ \text { date Feb 23 } 2007 & \text { dfrq } & 500.174\end{array}$

solvent $\mathrm{CDCl} 13 \mathrm{dn} \quad 500.176$

$\begin{aligned} \text { file } & \text { exp dpwr } \\ \text { ACQUISITION } & \text { dof }\end{aligned}$

$\begin{array}{lll}\text { ACQUISITION } & \\ \text { sfrq } & 500.176 & d m\end{array}$

$\begin{array}{lrlr}\mathrm{tn} & \mathrm{H} 1 & \mathrm{dmm} & \mathrm{dm} \\ \mathrm{at} & 2.048 & \mathrm{dmf} & 8770\end{array}$

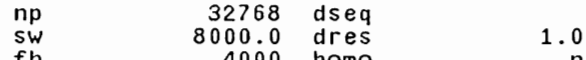

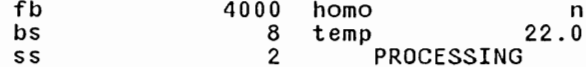

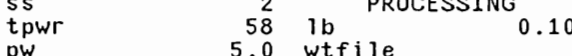

$\begin{array}{lrll}\mathrm{pw} & 5.0 & \mathrm{wtfi} & \\ \mathrm{d} 1 & 0 & \mathrm{proc} & \mathrm{ft} \\ \text { tof } & 0 & \mathrm{fn} & \text { not used }\end{array}$

$\begin{array}{lrl}\text { tof } & 0 & \text { fn } \\ \text { nt } & 10000 & \text { math used }\end{array}$

ct 64

$\begin{array}{lll}\text { a lock not used wexp } & \text { werr } \\ \text { gain } & & \end{array}$

i) FLAGS $n$ whs

in $n$

hs

sp DISPLAY -27.3

Wp $\quad 3684.1$

$\begin{array}{lr}\text { VS } & 127 \\ \text { SC } & 0 \\ \text { WC } & 250 \\ \text { hZmm } & 14.74\end{array}$

hZmm $\quad 14.74$

$\begin{array}{ll}\text { is } & 355.32 \\ \text { rf } 1 & 1499.0\end{array}$

th

ins cdc ph

1.000

wft
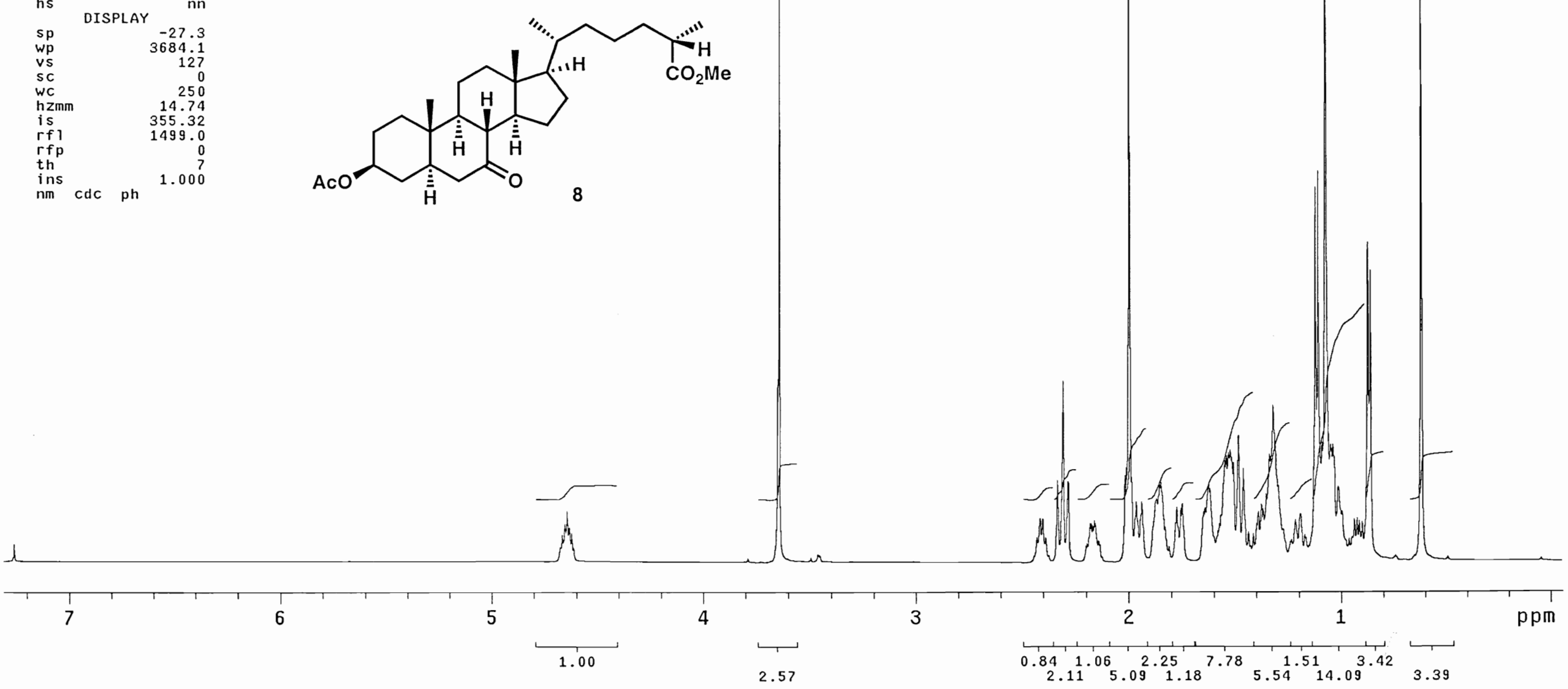
13C OBSERVE
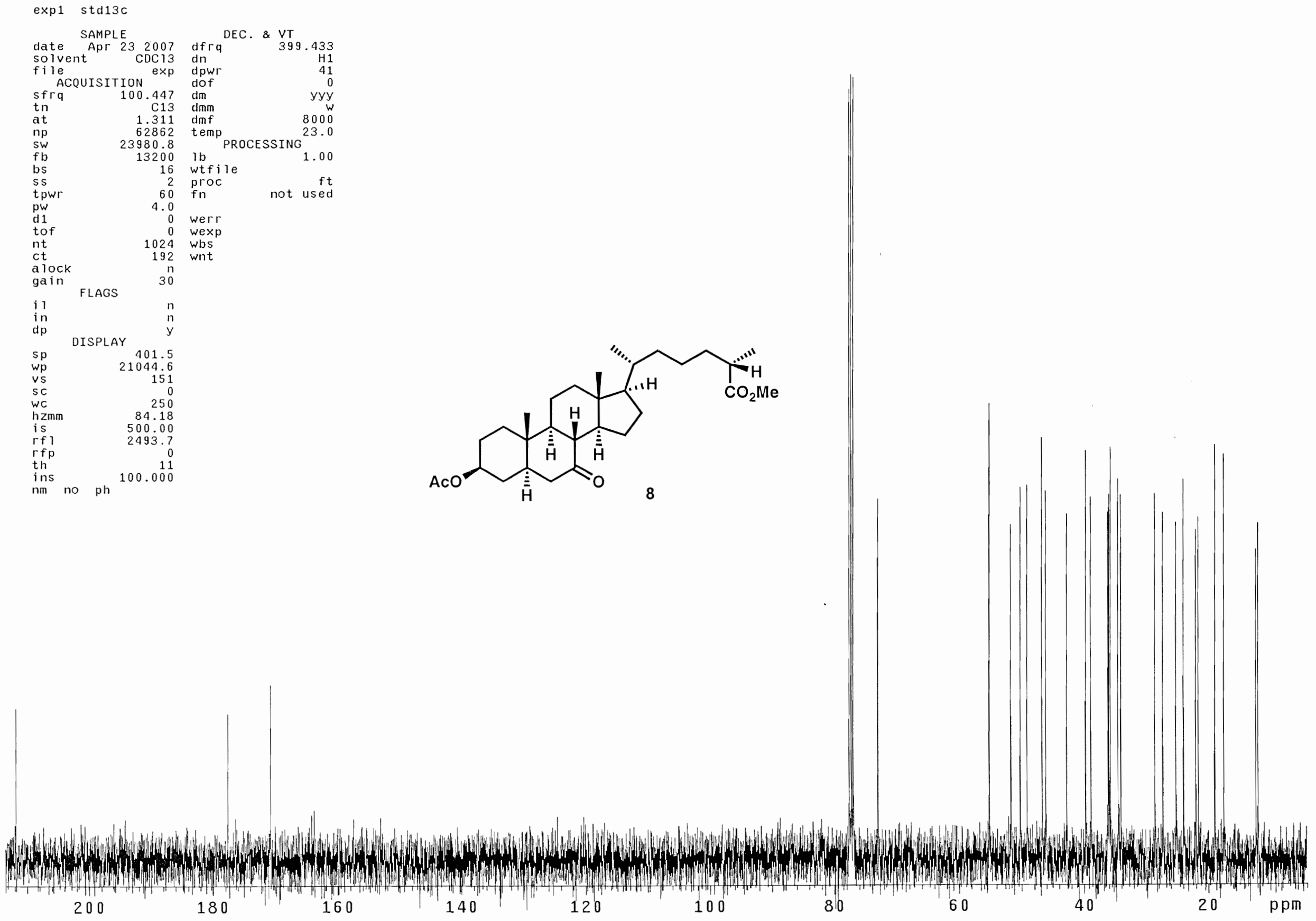
STANDARD PROTON PARAMETERS

\section{exp2 s2put}

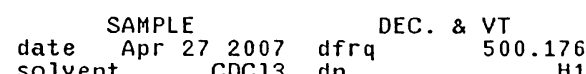

$\begin{array}{lrlr}\text { solvent } & \operatorname{CDCl} 13 & \text { dn } & \text { H1 } \\ \text { file } & \text { exp dpwr } & 32\end{array}$

$\begin{array}{rrrr}\text { ACQUISITION } & \text { dof } & 0 \\ \operatorname{sfrq} & 500.176 & \mathrm{dm} & \mathrm{nnn}\end{array}$

$\begin{array}{lrlr}s f r q & 500.176 & \mathrm{dm} & \mathrm{Hnn} \\ \mathrm{tn} & 2.041 & \mathrm{dmm} & \mathrm{c} \\ \mathrm{at} & 2.048 & \mathrm{dmf} & 8770\end{array}$

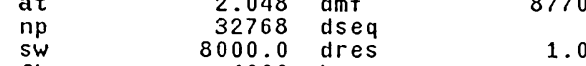

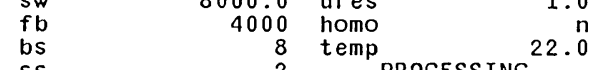

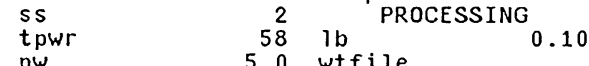

$\begin{array}{lrlr}\text { tpwr } & 5.0 & \text { wtfile } & \\ \text { pw } & 0 & \text { proc } & \text { ft } \\ \text { d1 } & 0 & \text { fn } & \text { not used } \\ \text { tof } & 1000 & \text { math } & \end{array}$

nt 1000 math $\quad 104$ not used

$\begin{array}{lrl}\text { ct } & 104 & \text { werr } \\ \text { alock } & \text { not wsed }\end{array}$

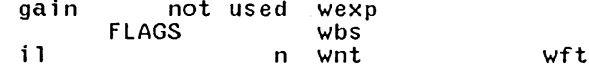

$\begin{array}{lll}i n & n & w n \\ d p & n & \end{array}$

hs DISPLAY nn

$\begin{array}{rr}\mathrm{sp} & 165.5 \\ w p & 3489.3\end{array}$

$\begin{array}{lr}\text { VS } & 12 \\ \text { SC } & 25 \\ \text { WC } & 13\end{array}$

$\begin{array}{lr}\text { hC } & 250 \\ \text { is } & 13.96 \\ \text { if } & 526.00\end{array}$

$\begin{array}{lr}r f f & 526.00 \\ r f p & 1499.0 \\ t h & 0\end{array}$

ins

$\mathrm{nm}$ cdc ph

1.000
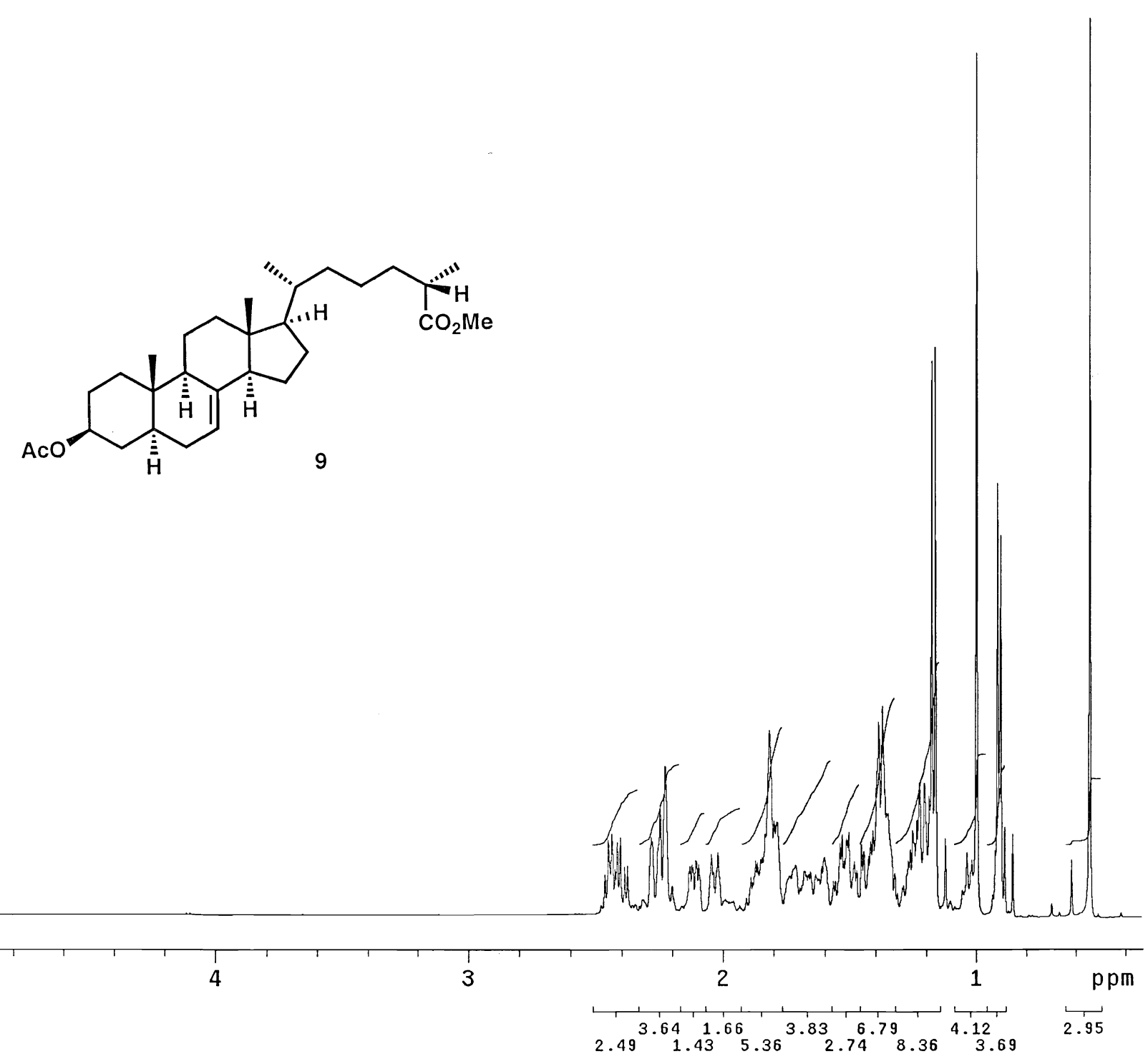
STANDARD CARBON PARAMETERS

exp2 s2pul

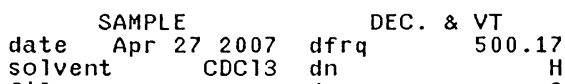

file exp dpwr $\quad 38$

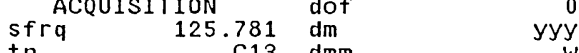

$\begin{array}{lrrr}\mathrm{tn} & \mathrm{C13} & \mathrm{dmm} & \mathrm{w} \\ \text { at } & 1.170 & \mathrm{dmf} & 15970\end{array}$

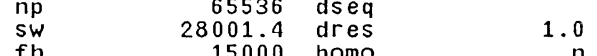

$\begin{array}{lrl}\text { bs } & 16 & \text { temp } \\ \text { tpwr } & 57 & \text { PROCESSING } \\ & 22.0\end{array}$

pw $\quad 8.0 \mathrm{lb} \quad 1.00$

$\begin{array}{lrll}\text { d1 } & 0.100 & \text { wtfile } & \\ \text { tof } & 0 & \text { proc } \\ \text { nt } & 1000 & \text { fn } & \text { ft }\end{array}$

$\begin{array}{lrl}\text { nt } & 1000 & \text { fn } \\ \text { ct } & 96 \text { math not used }\end{array}$

$\begin{array}{lrl}\text { alock } & \text { n } & \\ \text { gain } & 56 & \text { werr }\end{array}$

il FLAGS wexp

$\begin{array}{lll}\text { il } & n & \text { wbs } \\ \text { in } & n & \text { wnt }\end{array}$

hs nn

sp DISPLAY 768.0

$\begin{array}{rr}\text { Wp } & 22926.3 \\ \text { vS } & 62\end{array}$

$\begin{array}{ll}\text { SC } & 0 \\ \text { WC } & 250\end{array}$

hzmm $\quad 91.71$

$\begin{array}{ll}\text { is } & 500.00 \\ \text { rf } 1 & 205 i .1\end{array}$

$\begin{array}{ll}\mathrm{rfp} & 0 \\ \mathrm{th} & \end{array}$

ins cdc ph 100.000
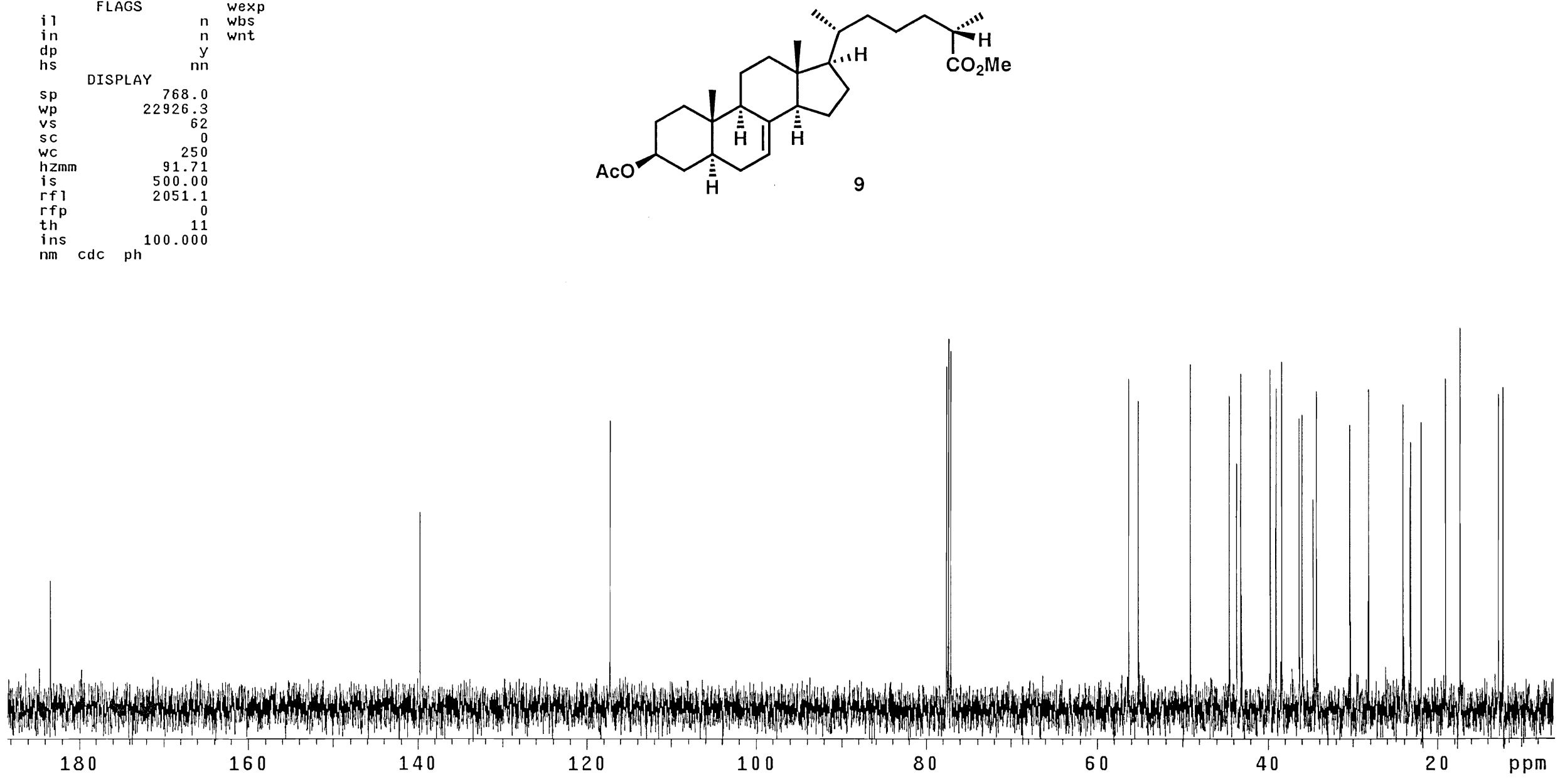
$\exp 1$ std1h

date $\begin{aligned} & \text { SAMPLE } \\ & \text { Jun } 52007 \text { dfrq DEC. \& }\end{aligned}$

$\begin{array}{lrlr}\text { date Jun } & 52007 & \text { dfrq } & 399.433 \\ \text { solvent } & \operatorname{CDCl3} & \text { dn } & H 1 \\ \text { file } & \text { exp } & \text { dpwr } & 43\end{array}$

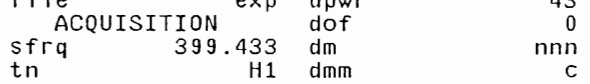

$\begin{array}{lrr}\text { at } & \text { H1 } & \text { dmm } \\ \text { at } & 2.731 & \text { dmf }\end{array}$

$\begin{array}{rrrr}\mathrm{np} & 32768 & \text { temp } & 12200 \\ \text { sw } & 5998.8 & & 23.0\end{array}$

$\begin{array}{lrl}\mathrm{SW} & 5998.8 & \mathrm{~b} \text { PROCESSING } \\ \mathrm{fb} & 3400 \quad \mathrm{~b} & 0.10\end{array}$

$\begin{array}{rrlr}\text { bs } & 8 & \text { wtfile } & \\ \text { ss } & 2 & \text { proc } & \mathrm{ft}\end{array}$

tpwr $\quad 59$ fn not used

pw 12.0 werr

$\begin{array}{lll}\text { di } & 0 & \text { werr } \\ \text { tof } & 0 & \text { wexp }\end{array}$

$\begin{array}{rrr}\text { nt } & 10000 & \text { wbs } \\ \mathrm{ct} & 80 & \text { wnt }\end{array}$

alock nain $n$
gain

FLAGS

in
$d p$

DISPLAY

$\begin{array}{rr}\mathrm{sp} & 0.0 \\ \mathrm{wp} & 3259.6 \\ \mathrm{vS} & 162\end{array}$

SC

$\begin{array}{lr}\text { WC } & 250 \\ \text { hzmm } & 13.04 \\ \text { is } & 240.88\end{array}$

$\begin{array}{ll}\text { is } & 240.88 \\ r f 1 & 1031.8\end{array}$

$\mathrm{rfp} \quad 0$

ins $\quad 1.000$

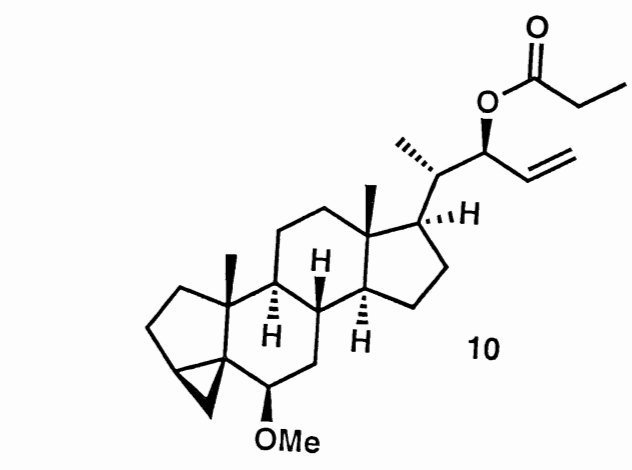

d

y

62
0
50

.8
0
20

(n) 
13C OBSERVE
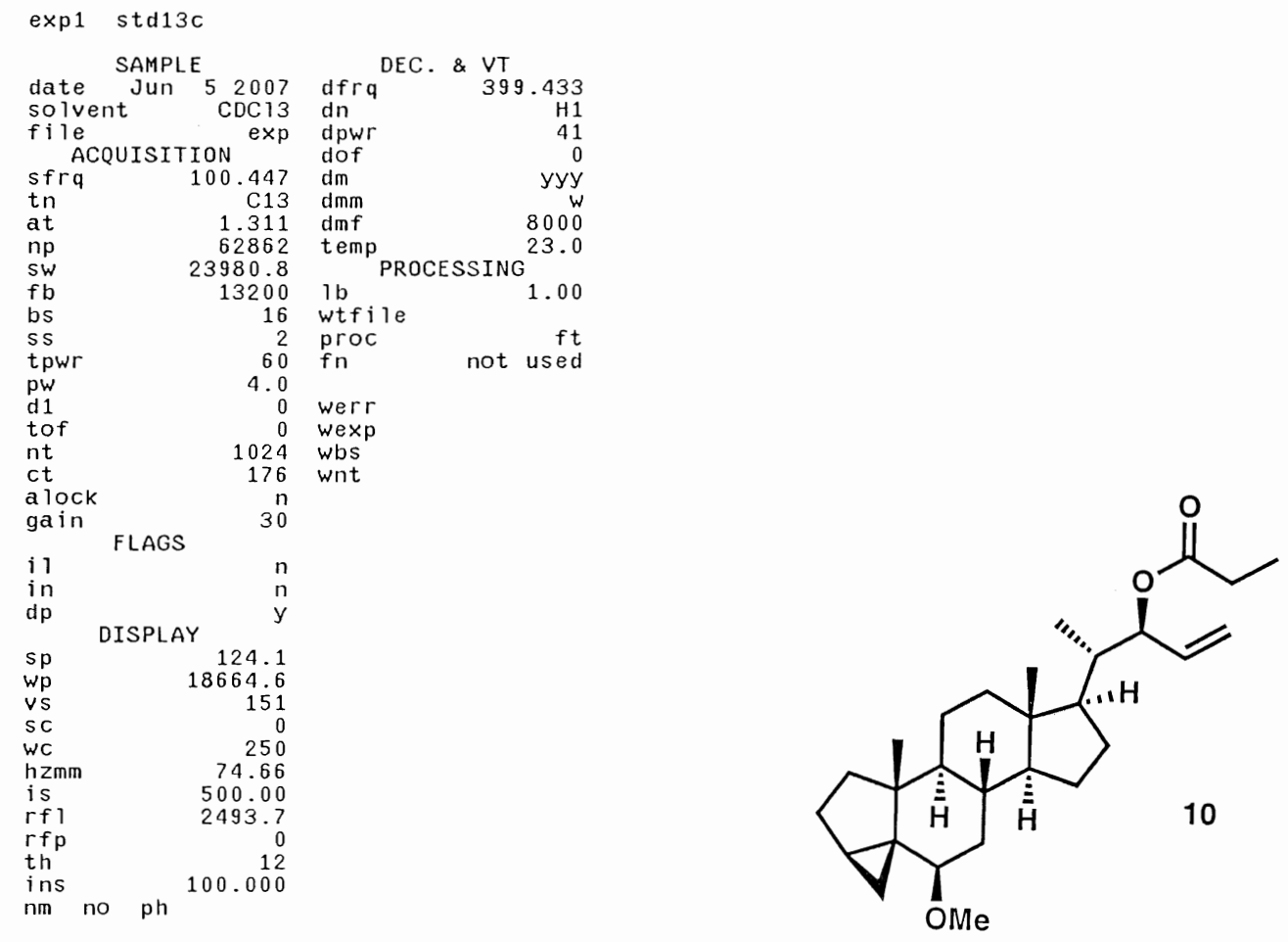

W. 
STANDARD PROTON PARAMETERS

exp2 S2puT
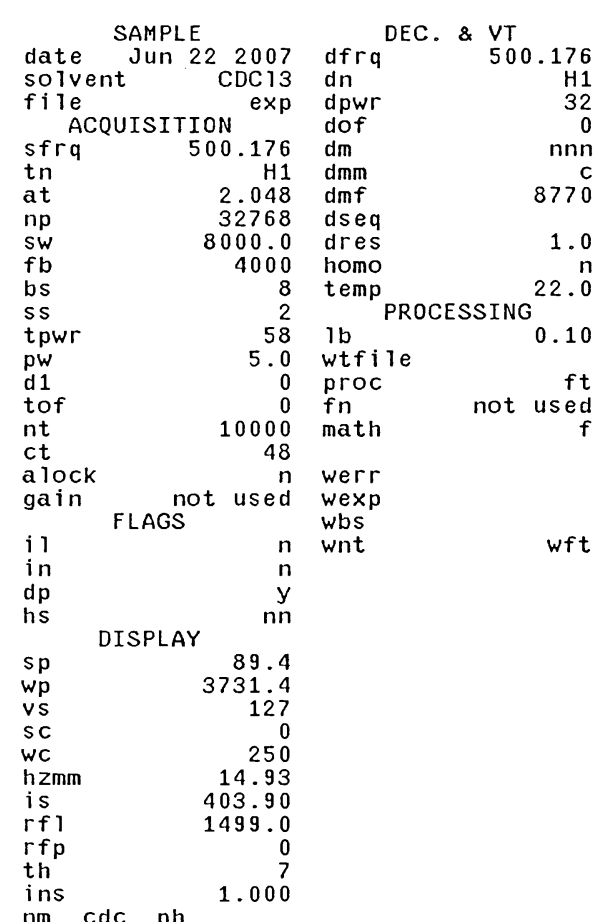

nm cdc ph

1.000
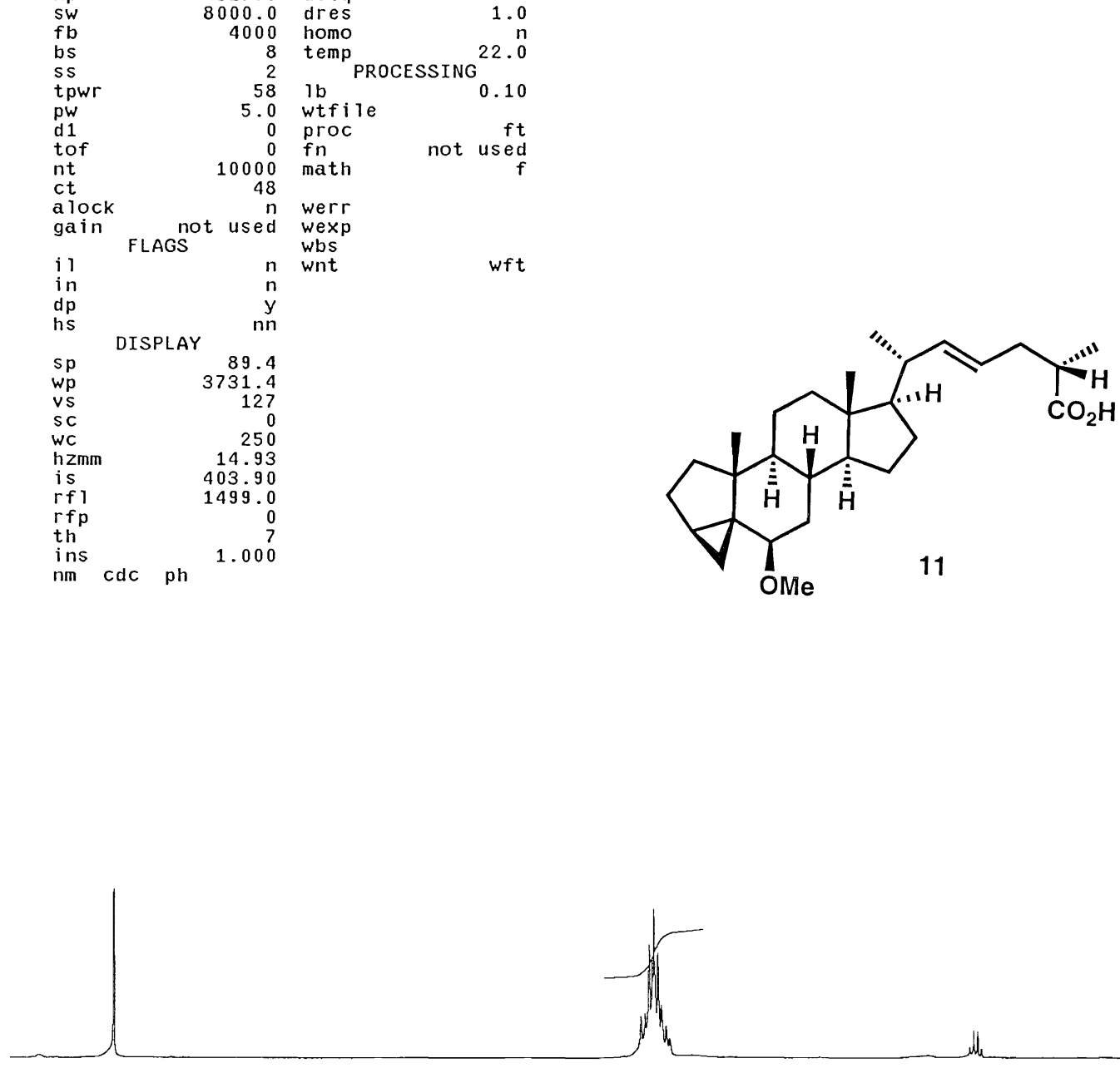

6

5

3

2.60

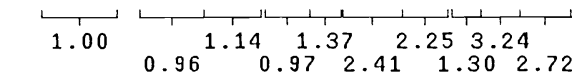

ppm 
13C OBSERVE
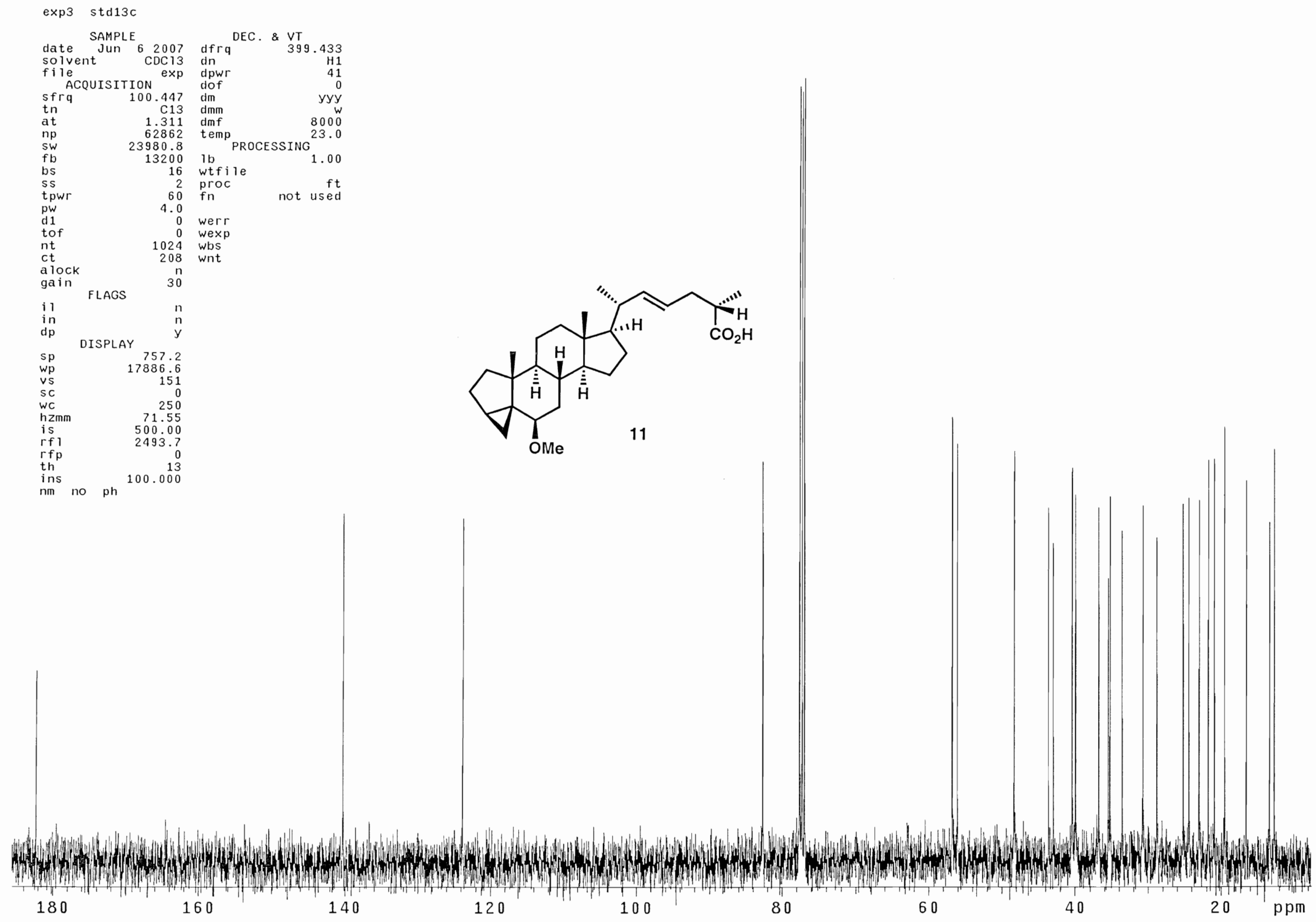
STANDARD 1 H OBSERVE

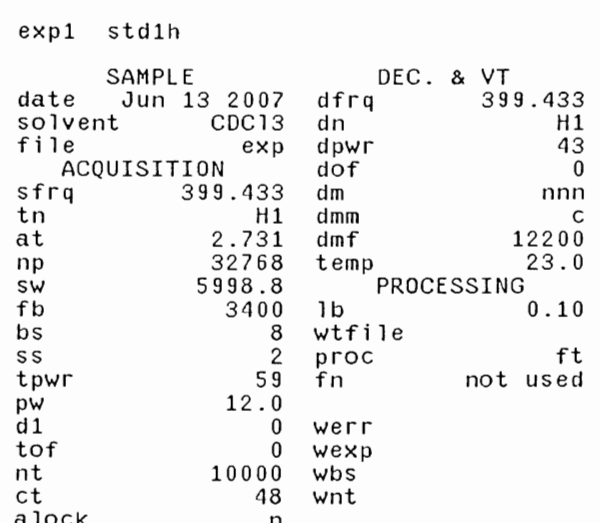

48 wht

gain not used

$\begin{array}{lr}\text { i } & n \\ \text { in } & n \\ \text { dp } & \text { DISPLAY } \\ \text { sp } & y \\ \text { wp } & 147.6 \\ \text { vs } & 2945.8 \\ \text { sc } & 108 \\ \text { wC } & 0 \\ \text { hZmm } & 250 \\ \text { is } & 11.78 \\ \text { rf } & 500.00 \\ \text { rfp } & 1031.8 \\ \text { th } & 0 \\ \text { ins } & 20 \\ \text { nm } & 1.000\end{array}$

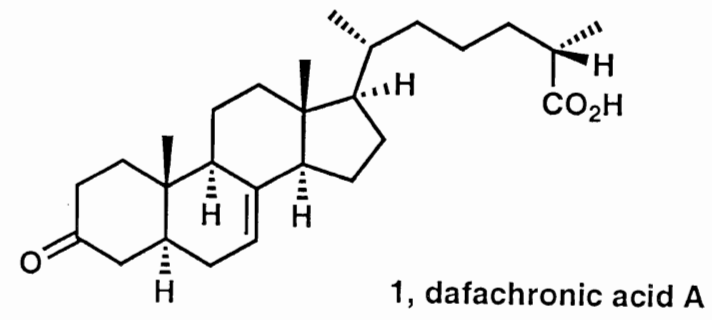

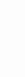

(
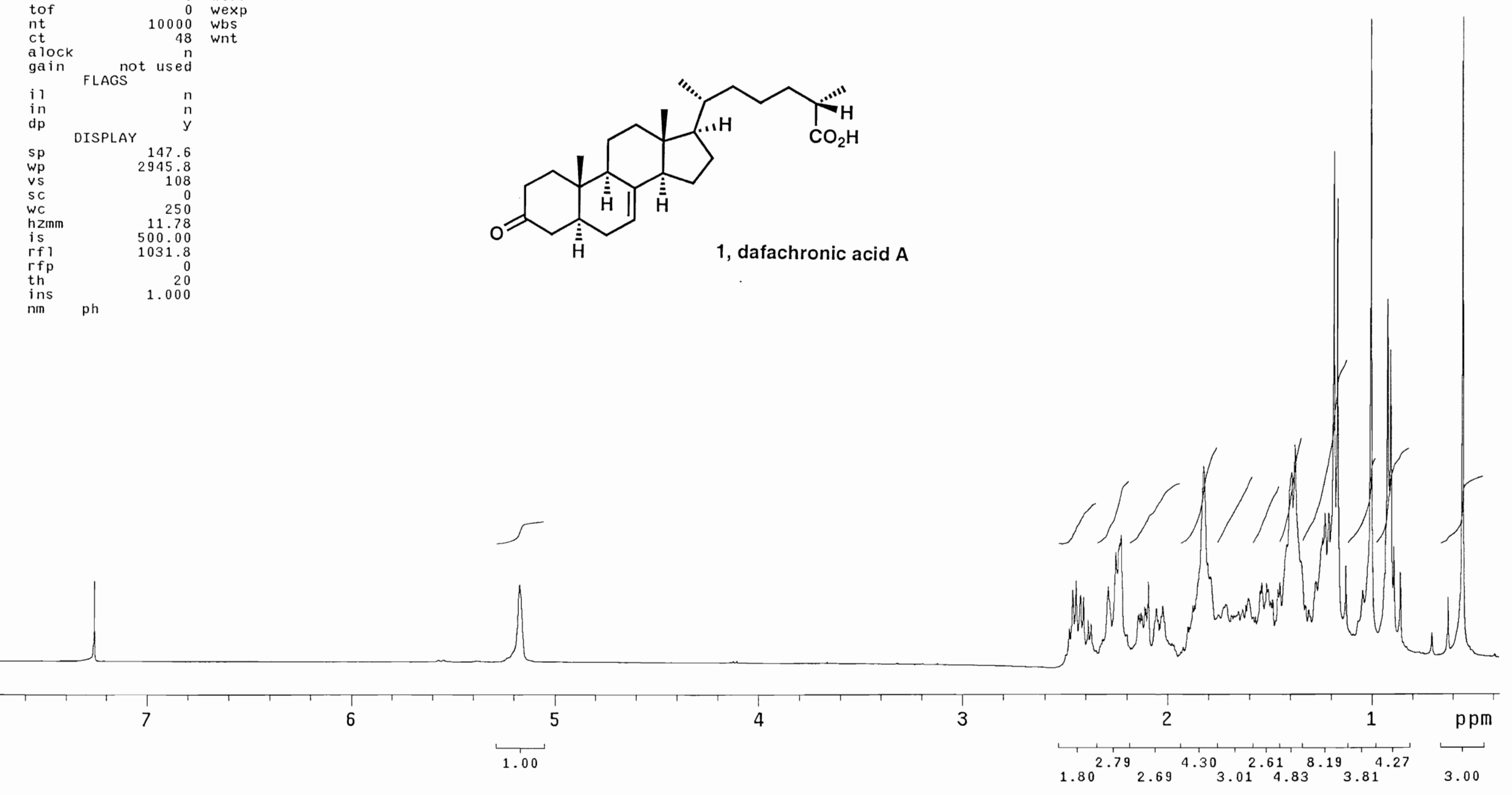
13C OBSERVE
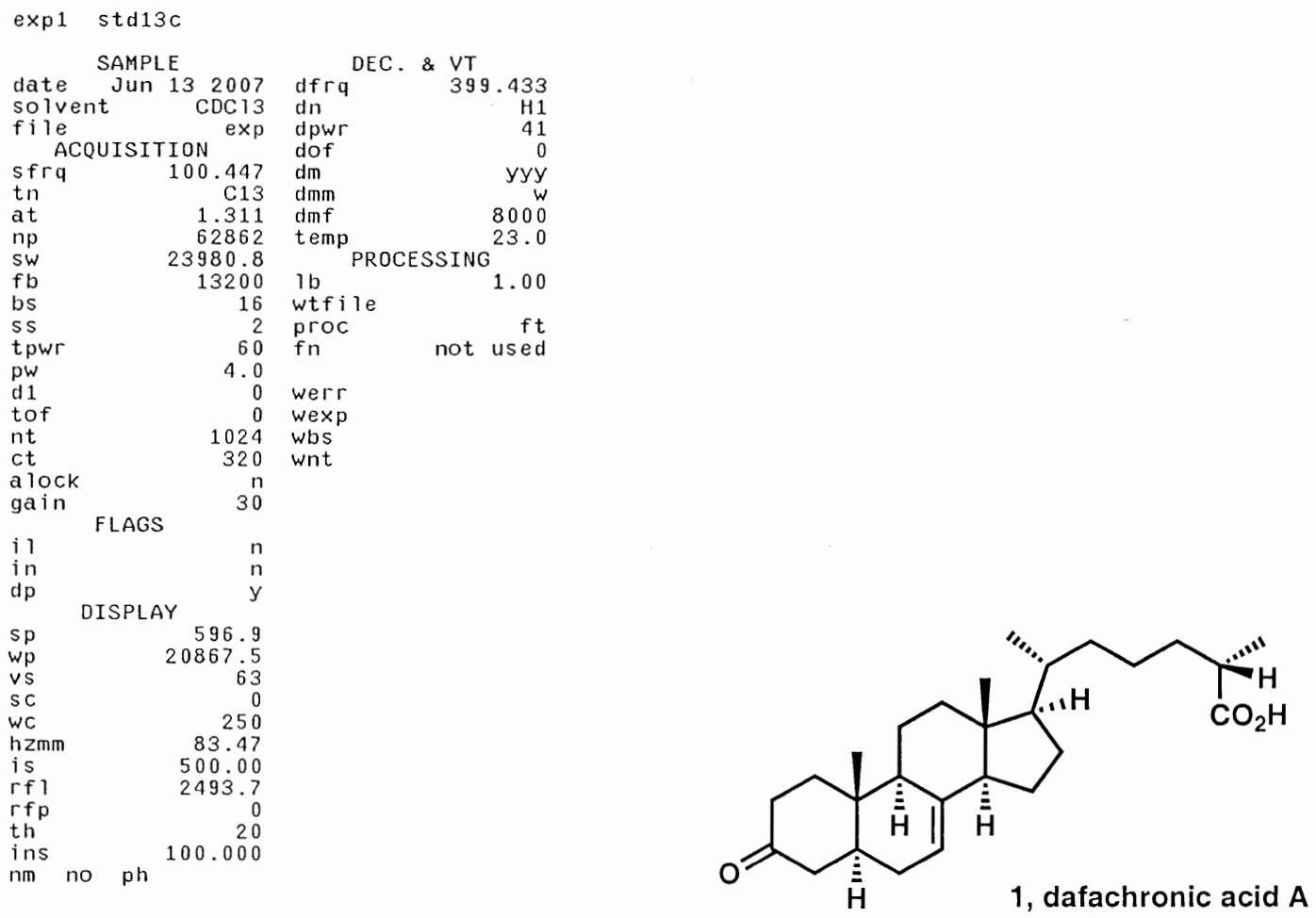

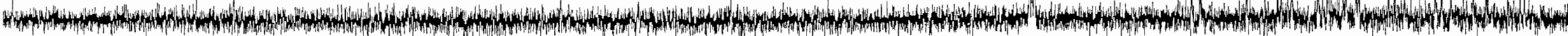

